\title{
Digital colorimetric analysis of the Turin Shroud
}

\author{
Christian Privitera \\ CRIS (Committee for Engineering Research on the Turin Shroud), via G.L. Bernini 108/1, Mira, Venice, Italy, \\ christian.privitera@gmail.com
}

\begin{abstract}
By using the data collected in previous colorimetric studies, a quantitative digital image with $\pm 4 \%$ uncertainty relative to the color values, has been made. This goal was achieved by colors correction of a digitalized photograph performed through comparison with some published measurements in CIE XYZ color space. Starting from that image construction, a simply color measurement method was performed by using a common photographic software, and consequently allowing the construction of a colorimetric database of the main observable details of the Turin Shroud (TS). With this tools some analysis on TS colors were performed and interesting results were found: for example, it has been quantitatively demonstrated that the background cloth colors, gradually and continuously changes toward the typical shades of body image. From this work it has been also possible to distinguish colors differences between blood stains and the other stains which have proved to have of different origins.
\end{abstract}

\section{Introduction}

The Turin Shroud (TS) is believed by many to be the burial cloth of Jesus of Nazareth when he had been put in a tomb in Palestine about 2000 years ago. TS has generated considerable controversy but, beyond all the innumerable widely debated questions, it is a matter of fact that the TS exists as an archaeological object: thus it can be directly and objectively observed [1]. The results of the studies carried out on the TS can be analyzed by scientific methods.

The TS is a linen sheet about $4.4 \mathrm{~m}$ long and $1.1 \mathrm{~m}$ wide, in which the complete front and back body images of a man are impressed. The cloth is hand-made and each yarn (diameter about $0.25 \mathrm{~mm}$ ) is composed of 70-200 linen fibers [2]. It has been shown by many scientists that the linen sheet enveloped the corpse of a man who had been scourged, crowned with thorns, crucified with nails, and stabbed by a lance in the side. Many other marks due to blood, fire, water and folding, are also impressed which have greatly damaged the double body image.

The TS has a front and a back image separated by a non-image zone of $0.18 \mathrm{~m}$; the images show an adult male, nude, well proportioned and muscular, with beard, mustache and long hair.

Many hypotheses and experimental tests have been carried out on linen fabrics to explain the formation of the body image, in favor of both authenticity and the other way around [2]. Despite some macroscopic results with resemblance to the TS image which have often been achieved limited to the face, none of them has come close to reproduce all the TS image characteristics. In other words, even if at first sight, many image copies are similar to that of the TS, until now no experimental test has been able to reproduce all the characteristics found on the original. 
From this brief description it is easy to understand how studies on the TS must be conducted wideranging in many fields of human knowledge, but given the current difficulty to directly perform analysis on the Relic, the studies are performed on materials collected by the STURP (Shroud of Turin Research Project) in 1978 and during other rare occasions.

Colorimetric analysis can be a way to solve some questions, but the works on this research field are few and too approximate because they have been performed on not calibrated image or didn't use a repeatable measurement method.

To perform colorimetric analysis a calibrated quantitative image of the TS was built and a standard measurements procedure has been determined on it [3,4]: with these tools many researchers will be able to perform colorimetric analysis on TS. This paper integrates with new content what has been already published [4].

\section{Measurement instrument and procedure}

\subsection{The quantitative image}

The aforementioned image was created starting from an image taken by photographer G. Durante in 2002. This picture with sizes of $6996 \times 24197$ pixel with a resolution of $300 \mathrm{dpi}$ was used as the base image.

In 1978 researchers Soardo et al. performed color measurements on various details of the TS and expressed the results in CIE XYZ color space [5].

For calibrating the Durante's image, 18 points were chosen on it with a certain correspondence to the measurements points reported in Soardo's paper. By using a circular mask of a common photographic software with a diameter of 68 pixel (corresponding to the $13 \mathrm{~mm}$ of Soardo's colorimeter sampling spot) the statistical values of the single RGB color channel (values range 0 255) were read by means of the histogram function.

The color data, acquired by using Paint Shop ${ }^{\mathrm{TM}}$ Pro $\subset$, were converted from their sRGB color space representation to CIE XYZ space [6] and the CIE D55 type illuminant (used by G.Durante to realize his photographic work) was converted into CIE A type (used by Soardo et al. for colorimetric measurements) by using the Bradford matrix [7]. The acquired RGB values were converted by the application of the followings matrix operations (subscript A and D55 identify color values expressed in CIE type A and type D55 illuminant):

$$
\left[\begin{array}{l}
X \\
Y \\
Z
\end{array}\right]_{A}=\left[T_{1}\right] \cdot\left[T_{2}\right] \cdot\left[\begin{array}{l}
R \\
G \\
B
\end{array}\right]_{D 55}=[T] \cdot\left[\begin{array}{l}
R \\
G \\
B
\end{array}\right]_{D 55}
$$

Where:

$$
\left[T_{1}\right]=\left[\begin{array}{ccc}
1.1803 & 0.0969 & -0.1386 \\
0.1334 & 0.9183 & -0.0499 \\
-0.0217 & 0.0327 & 0.3732
\end{array}\right] ; \quad\left[T_{2}\right]=\left[\begin{array}{ccc}
0.4125 & 0.3576 & 0.1804 \\
0.2127 & 0.7152 & 0.0722 \\
0.0193 & 0.1192 & 0.9503
\end{array}\right] ; \quad[T]=\left[\begin{array}{lll}
0.5049 & 0.4748 & 0.0882 \\
0.2494 & 0.6985 & 0.0429 \\
0.0052 & 0.0601 & 0.3531
\end{array}\right]
$$

- [T1] is the Bradford matrix for illuminant conversion from CIE D55 to CIE type A;

- [T2] is the conversion matrix from sRGB to CIE XYZ color space;

- [T] is the transformation matrix, the resulting matrix product of [T1] and [T2].

The $\mathrm{x}, \mathrm{y}, \mathrm{z}$ chromatic coordinates (values range $0-1$ ) can be obtained by using eq. 3 :

$$
x=\frac{X}{X+Y+Z} ; \quad y=\frac{Y}{X+Y+Z} ; \quad z=\frac{Z}{X+Y+Z}
$$


The acquired data were compared with the Soardo's measurements, finding that the mean differences were caused by an additive systematic effect. So additional constants $\delta_{\mathrm{x}}, \delta_{\mathrm{y}}, \delta_{\mathrm{z}}$ were calculated by the difference between the arithmetic mean of the 18 converted RGB data acquired on Durante's photography and the corresponding arithmetic mean of the 18 reference measurements made by Soardo. The calculated constants $\delta_{\mathrm{x}}, \delta_{\mathrm{y}}, \delta_{\mathrm{z}}$ have been added algebraically to the colorimetric values obtained by conversion of the RGB values to eliminate the additive systematic effect (Fig. 1 and 2).

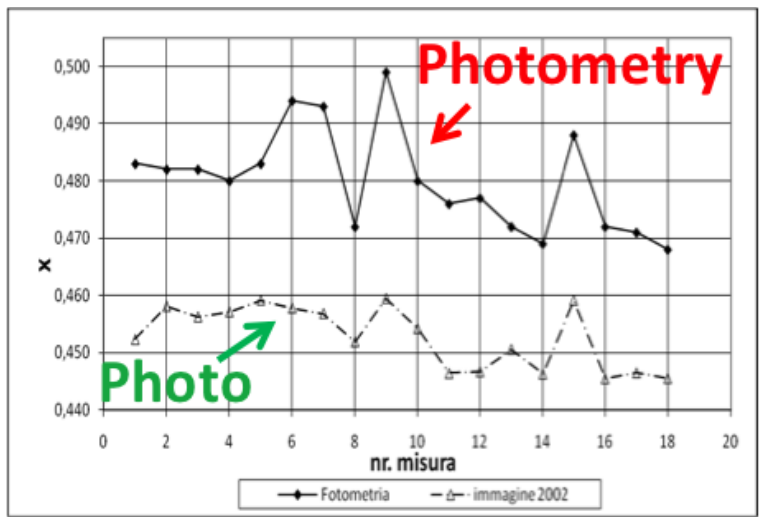

Figure 1. X coordinate value before correction. It can be seen the addictive systematic effect.

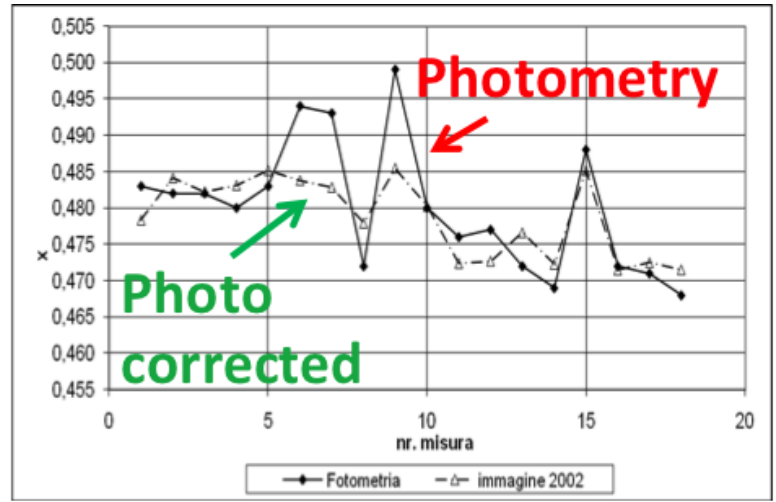

Figure 2. $\mathrm{X}$ coordinate value after correction with the additional constant.

After colors correction in CIE XYZ color space, the image was reconverted to sRGB standard by using the inverse color space transformation. The image built in such a manner turned out to be characterized by a calculated uncertainty on color values of $\pm 4 \%$.

\subsection{Measurement instrument and method}

It was determined a measuring procedure starting from the method used for the quantitative image construction. As was done for the image calibration, the measurement instrument was "built" with masks and histogram functions of a common photographic software (Fig.3), through which were possible to read out the R, G, B channel value of a determinate area: the shape and the dimension of that area are fundamental elements, so it was important to investigate it.

The shape of the chosen selector was the circle, first of all for its wide availability in all the software's mask lists, then for its curved contours and because only center coordinates are needed for positioning it. 


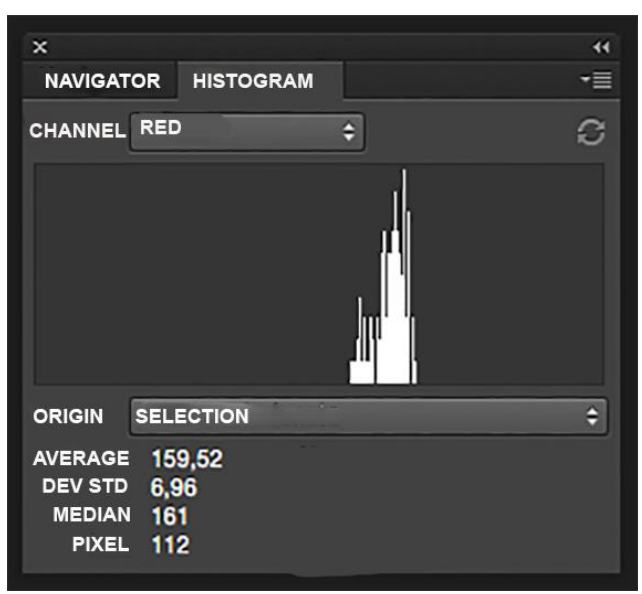

Figure 3. Histogram function of common photographic software. With this tool it can be readed the average value of a single color channel in selected area

The size of the sampling area was determined by evaluating:

the amount of pixels on which the program executes the average values of the individual channels, because it determines the sensitivity of the variations; the capability in balancing the tonal differences derived from the weft of the fabric. In fact the TS image is composed by an alternance of clear and dark fibers, so for balancing this effect the dimension of the measurement area must include equal number of fibers; the capability to test very small details.

The diameter of the circular mask was chosen equal to 12 pixels (in metric scale $\mathrm{d}=2.3 \mathrm{~mm}$ ) (Fig.4).

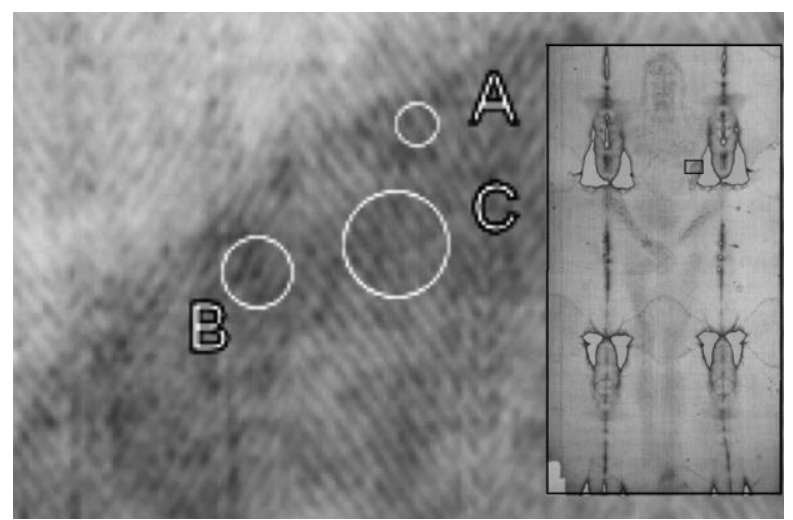

Figure 4. Shape and size of the sampling area used in the image processing. Diameter dimension comparison: A) 12 pixel B) 20 pixel C) 40 pixel.

Robustness analysis was performed in order to verify the stability of the RGB measurements to variation of the shape and the dimension area of the measurement mask.

Original image was splitted in two parts (front and back image) with precise dimension. Then, for each part a coordinate system was defined (Fig. 5): (h, k) coordinates corresponding to the pixel position, so they can be detected directly from the software used for measurements.

The color measurement procedure can be summarized as follows: to open the histogram function, to choose a circular selection mask with 12 pixel diameter, to apply the mask on selected features, to read the RGB values by means values using the histogram function. 
The measurement method can be applied to the Durante's photography by using a data-sheet for color conversion to obtain $\mathrm{XYZ}$ colors values and then to compare the results with the follow database.
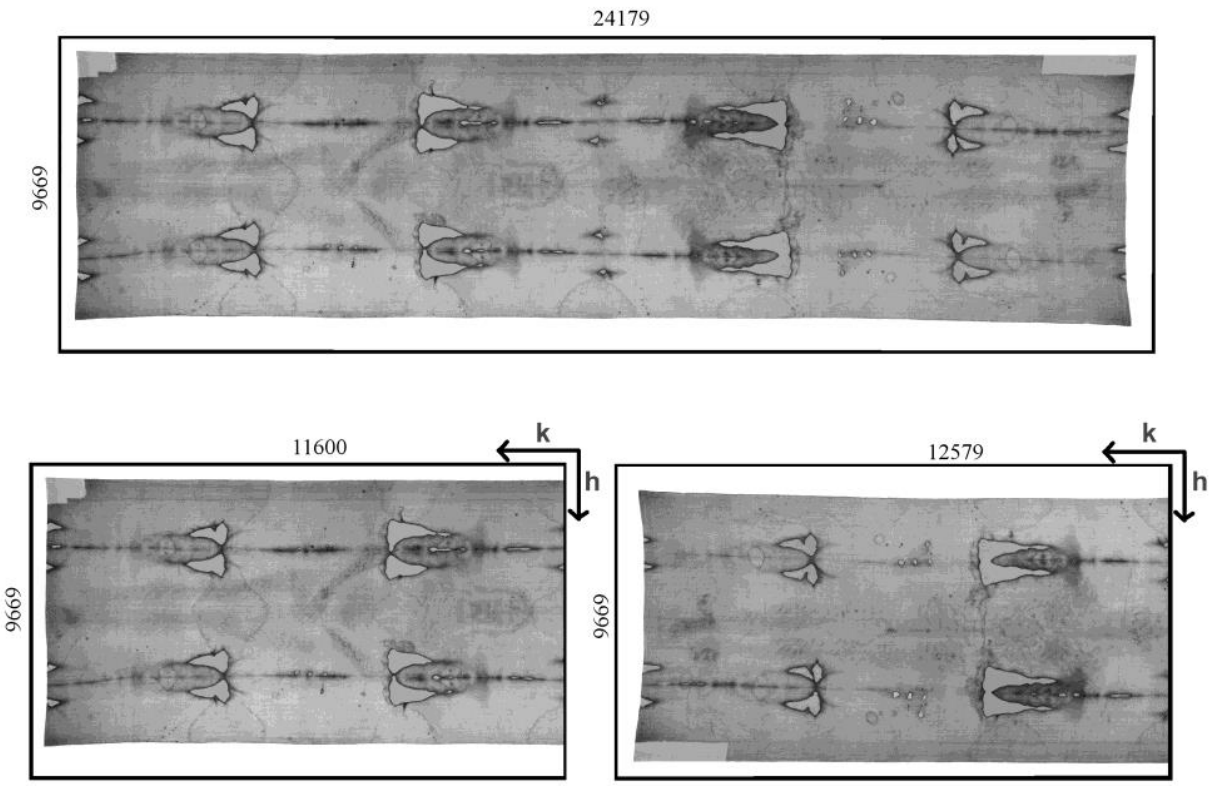

Figure 5. The splitted image and the (h, k) coordinate system origin corner and axis orientation or for measurements.

\section{Colorimetric analysis of details}

\subsection{Colorimetric measurements}

Once calibrated the quantitative image, the following measurement has been performed on it: 54 samples both on the front side and the dorsal of the body image (Fig. 6-7 and tab. 1-2); 54 samples both on the front side and the dorsal of the bloodstains (Fig. 8-9 and tab. 3-4); 54 samples both on the front side and the dorsal of the background cloth (Fig. 10-11 and tab. 5-6); 30 samples, 16 on the front side and 14 on the dorsal side of the water stains areas (Fig. 12-13 and tab. 7-8);

30 samples, 16 on the front side and 14 on the dorsal side of the burns areas (Fig. 12-13 and tab. 7-8).

For database and plots, chromatic coordinates in CIE XYZ color space were used because they are directly comparable with photometric measurements. So from the tables, for each measurement point reported in figures, the coordinates of the center of the sampling area $(\mathrm{h}, \mathrm{k})$, the chromatic coordinates obtained from RGB channel values conversion $(\mathrm{x}, \mathrm{y}, \mathrm{z})$ and the dimensionless chromatic ratios used also for the plots construction $(\mathrm{x} / \mathrm{y}, \mathrm{x} / \mathrm{z}, \mathrm{y} / \mathrm{z})$ can be readed.

In table 9 the values range and their arithmetical average of the analyzed TS features were reported. 


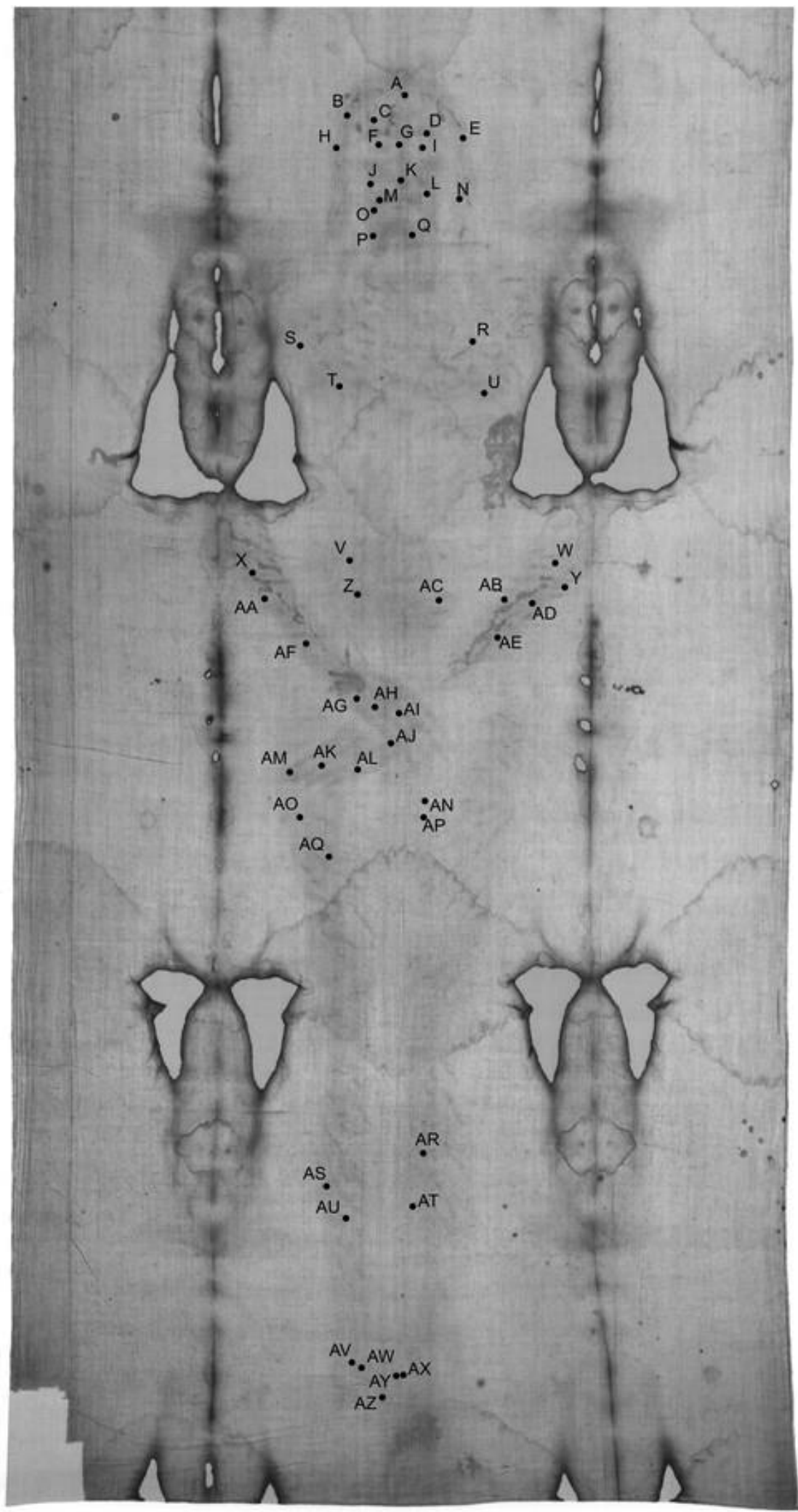

Figure 6. Measurement points on BODY IMAGE front. 
Table 1. Reference table of the position in the (h, k) coordinate system of the measurement points in fig. 6 and related color values and dimensionless ratios.

\begin{tabular}{|c|c|c|c|c|c|c|c|c|}
\hline \multicolumn{9}{|c|}{ BODY IMAGE chromatic values FRONT } \\
\hline & $\mathbf{h}$ & $\mathbf{k}$ & $x$ & $y$ & $z$ & $x / y$ & $x / z$ & $y / z$ \\
\hline $\mathbf{A}$ & 3305 & 662 & 0.48 & 0.42 & 0.10 & 1.14 & 4.87 & 4.26 \\
\hline B & 2877 & 805 & 0.48 & 0.42 & 0.10 & 1.14 & 4.72 & 4.16 \\
\hline $\mathbf{C}$ & 3097 & 846 & 0.48 & 0.42 & 0.10 & 1.14 & 4.74 & 4.17 \\
\hline D & 3472 & 945 & 0.49 & 0.42 & 0.09 & 1.15 & 5.23 & 4.56 \\
\hline $\mathbf{E}$ & 3730 & 966 & 0.48 & 0.42 & 0.11 & 1.13 & 4.55 & 4.02 \\
\hline $\mathbf{F}$ & 3280 & 1038 & 0.48 & 0.42 & 0.10 & 1.14 & 4.73 & 4.17 \\
\hline G & 3122 & 1039 & 0.48 & 0.42 & 0.10 & 1.14 & 4.71 & 4.14 \\
\hline $\mathbf{H}$ & 2806 & 1059 & 0.48 & 0.42 & 0.10 & 1.13 & 4.66 & 4.11 \\
\hline I & 3449 & 1059 & 0.48 & 0.42 & 0.10 & 1.13 & 4.80 & 4.24 \\
\hline $\mathbf{J}$ & 3057 & 1300 & 0.48 & 0.42 & 0.10 & 1.14 & 4.79 & 4.22 \\
\hline $\mathbf{K}$ & 3286 & 1317 & 0.49 & 0.42 & 0.09 & 1.15 & 5.25 & 4.56 \\
\hline $\mathbf{L}$ & 3504 & 1393 & 0.48 & 0.42 & 0.10 & 1.13 & 4.78 & 4.22 \\
\hline $\mathbf{M}$ & 3119 & 1426 & 0.48 & 0.42 & 0.10 & 1.15 & 5.10 & 4.45 \\
\hline $\mathbf{N}$ & 3714 & 1438 & 0.48 & 0.42 & 0.10 & 1.13 & 4.71 & 4.16 \\
\hline $\mathbf{O}$ & 3088 & 1508 & 0.48 & 0.42 & 0.10 & 1.14 & 4.91 & 4.30 \\
\hline $\mathbf{P}$ & 3088 & 1700 & 0.49 & 0.42 & 0.09 & 1.15 & 5.25 & 4.57 \\
\hline $\mathbf{Q}$ & 3371 & 1711 & 0.49 & 0.42 & 0.09 & 1.15 & 5.18 & 4.51 \\
\hline $\mathbf{R}$ & 3821 & 2482 & 0.48 & 0.42 & 0.10 & 1.13 & 4.69 & 4.14 \\
\hline $\mathbf{S}$ & 2541 & 2539 & 0.48 & 0.42 & 0.10 & 1.13 & 4.75 & 4.19 \\
\hline $\mathbf{T}$ & 2830 & 2824 & 0.48 & 0.42 & 0.10 & 1.13 & 4.58 & 4.05 \\
\hline $\mathbf{U}$ & 3862 & 2845 & 0.48 & 0.42 & 0.10 & 1.13 & 4.73 & 4.17 \\
\hline $\mathbf{V}$ & 2884 & 4121 & 0.48 & 0.42 & 0.10 & 1.13 & 4.70 & 4.17 \\
\hline $\mathbf{W}$ & 4413 & 4188 & 0.48 & 0.42 & 0.10 & 1.13 & 4.60 & 4.06 \\
\hline $\mathbf{X}$ & 2183 & 4224 & 0.48 & 0.42 & 0.10 & 1.14 & 4.68 & 4.11 \\
\hline $\mathbf{Y}$ & 4513 & 4349 & 0.48 & 0.42 & 0.10 & 1.13 & 4.62 & 4.08 \\
\hline$Z$ & 2943 & 4367 & 0.48 & 0.42 & 0.10 & 1.14 & 4.93 & 4.32 \\
\hline $\mathbf{A A}$ & 2257 & 4400 & 0.48 & 0.42 & 0.10 & 1.14 & 4.83 & 4.24 \\
\hline AB & 4051 & 4438 & 0.48 & 0.42 & 0.10 & 1.13 & 4.64 & 4.11 \\
\hline $\mathbf{A C}$ & 3582 & 4452 & 0.48 & 0.42 & 0.10 & 1.13 & 4.63 & 4.09 \\
\hline AD & 4241 & 4460 & 0.48 & 0.42 & 0.10 & 1.13 & 4.64 & 4.11 \\
\hline $\mathbf{A E}$ & 4009 & 4695 & 0.48 & 0.42 & 0.10 & 1.13 & 4.68 & 4.13 \\
\hline $\mathbf{A F}$ & 2587 & 4751 & 0.48 & 0.42 & 0.10 & 1.14 & 4.74 & 4.18 \\
\hline AG & 2906 & 5173 & 0.48 & 0.42 & 0.10 & 1.13 & 4.77 & 4.21 \\
\hline $\mathbf{A H}$ & 3087 & 5233 & 0.48 & 0.42 & 0.10 & 1.14 & 4.89 & 4.28 \\
\hline AI & 3274 & 5271 & 0.48 & 0.42 & 0.10 & 1.14 & 4.81 & 4.22 \\
\hline $\mathbf{A J}$ & 3220 & 5482 & 0.48 & 0.42 & 0.10 & 1.14 & 4.83 & 4.25 \\
\hline AK & 2685 & 5662 & 0.48 & 0.42 & 0.10 & 1.14 & 4.74 & 4.18 \\
\hline $\mathbf{A L}$ & 2978 & 5689 & 0.48 & 0.42 & 0.10 & 1.13 & 4.82 & 4.25 \\
\hline $\mathbf{A M}$ & 2453 & 5692 & 0.48 & 0.42 & 0.10 & 1.14 & 4.78 & 4.20 \\
\hline AN & 3484 & 5922 & 0.48 & 0.42 & 0.10 & 1.13 & 4.69 & 4.15 \\
\hline AO & 2528 & 6016 & 0.48 & 0.42 & 0.10 & 1.13 & 4.67 & 4.11 \\
\hline AP & 3447 & 6059 & 0.48 & 0.42 & 0.11 & 1.13 & 4.53 & 4.01 \\
\hline AQ & 2744 & 6346 & 0.48 & 0.42 & 0.10 & 1.14 & 4.88 & 4.29 \\
\hline $\mathbf{A R}$ & 3436 & 8545 & 0.48 & 0.42 & 0.10 & 1.13 & 4.79 & 4.22 \\
\hline $\mathbf{A S}$ & 2734 & 8801 & 0.48 & 0.42 & 0.10 & 1.14 & 4.79 & 4.22 \\
\hline AT & 3377 & 8950 & 0.48 & 0.42 & 0.10 & 1.13 & 4.74 & 4.19 \\
\hline $\mathbf{A U}$ & 2889 & 9033 & 0.48 & 0.42 & 0.10 & 1.13 & 4.81 & 4.25 \\
\hline AV & 2936 & 10122 & 0.48 & 0.42 & 0.11 & 1.13 & 4.55 & 4.02 \\
\hline $\mathbf{A W}$ & 2992 & 10140 & 0.48 & 0.42 & 0.10 & 1.14 & 4.67 & 4.11 \\
\hline $\mathbf{A X}$ & 3285 & 10207 & 0.48 & 0.42 & 0.10 & 1.13 & 4.62 & 4.08 \\
\hline AY & 3274 & 10221 & 0.48 & 0.42 & 0.10 & 1.13 & 4.63 & 4.09 \\
\hline $\mathbf{A Z}$ & 3148 & 10379 & 0.48 & 0.42 & 0.10 & 1.13 & 4.70 & 4.15 \\
\hline
\end{tabular}


MATEC Web of Conferences

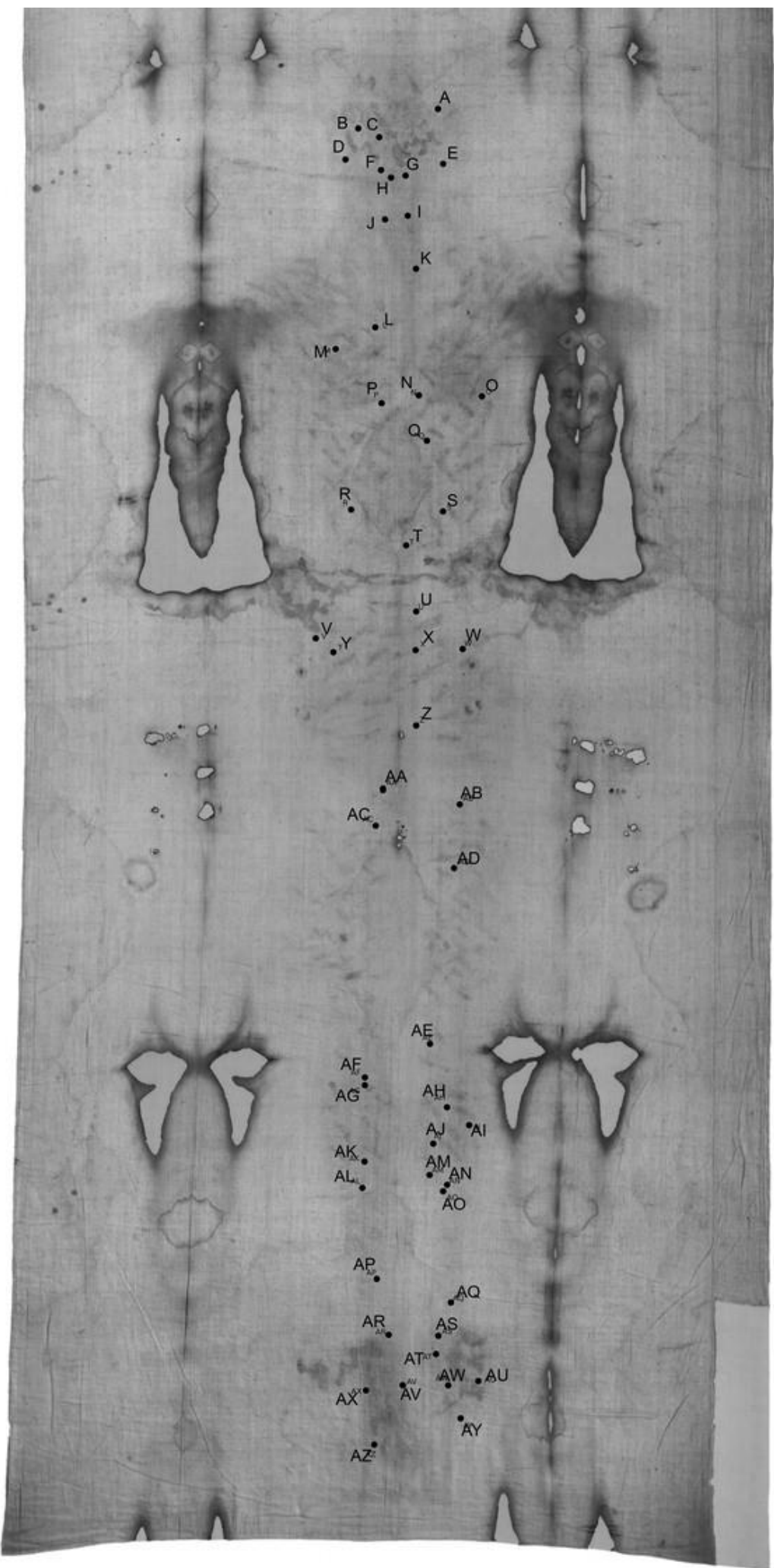

Figure 7. Measurement points on BODY IMAGE front. 
Table 2. Reference table of the position in the (h, k) coordinate system of the measurement points in fig. 7 and related color values and dimensionless ratios.

\begin{tabular}{|c|c|c|c|c|c|c|c|c|}
\hline \multicolumn{9}{|c|}{ BODY IMAGE chromatic values BACK } \\
\hline & $\mathbf{h}$ & $\mathbf{k}$ & $x$ & $y$ & $z$ & $x / y$ & $x / z$ & $y / z$ \\
\hline $\mathbf{A}$ & 3997 & 783 & 0.48 & 0.42 & 0.10 & 1.14 & 4.92 & 4.30 \\
\hline B & 3373 & 935 & 0.48 & 0.42 & 0.10 & 1.13 & 4.77 & 4.22 \\
\hline $\mathbf{C}$ & 3543 & 1007 & 0.48 & 0.42 & 0.10 & 1.14 & 5.06 & 4.44 \\
\hline D & 3277 & 1179 & 0.48 & 0.42 & 0.10 & 1.13 & 4.75 & 4.19 \\
\hline $\mathbf{E}$ & 4040 & 1209 & 0.48 & 0.42 & 0.10 & 1.13 & 4.57 & 4.05 \\
\hline $\mathbf{F}$ & 3548 & 1266 & 0.48 & 0.42 & 0.10 & 1.13 & 4.89 & 4.31 \\
\hline $\mathbf{G}$ & 3745 & 1299 & 0.48 & 0.42 & 0.10 & 1.14 & 4.91 & 4.32 \\
\hline $\mathbf{H}$ & 3633 & 1315 & 0.48 & 0.42 & 0.09 & 1.14 & 5.11 & 4.48 \\
\hline I & 3762 & 1627 & 0.48 & 0.42 & 0.11 & 1.13 & 4.54 & 4.00 \\
\hline $\mathbf{J}$ & 3581 & 1650 & 0.48 & 0.42 & 0.10 & 1.13 & 4.75 & 4.20 \\
\hline $\mathbf{K}$ & 3824 & 2028 & 0.48 & 0.42 & 0.10 & 1.14 & 4.61 & 4.07 \\
\hline $\mathbf{L}$ & 3515 & 2488 & 0.48 & 0.42 & 0.10 & 1.13 & 4.67 & 4.13 \\
\hline M & 3202 & 2665 & 0.48 & 0.42 & 0.10 & 1.14 & 4.58 & 4.03 \\
\hline $\mathbf{N}$ & 3853 & 3018 & 0.48 & 0.42 & 0.11 & 1.13 & 4.48 & 3.97 \\
\hline O & 4339 & 3025 & 0.48 & 0.42 & 0.11 & 1.13 & 4.54 & 4.00 \\
\hline $\mathbf{P}$ & 3560 & 3074 & 0.47 & 0.42 & 0.11 & 1.13 & 4.48 & 3.97 \\
\hline $\mathbf{Q}$ & 3920 & 3372 & 0.47 & 0.42 & 0.11 & 1.13 & 4.42 & 3.91 \\
\hline $\mathbf{R}$ & 3316 & 3902 & 0.47 & 0.42 & 0.11 & 1.13 & 4.41 & 3.91 \\
\hline $\mathbf{S}$ & 4040 & 3921 & 0.48 & 0.42 & 0.11 & 1.13 & 4.49 & 3.97 \\
\hline $\mathbf{T}$ & 3752 & 4190 & 0.47 & 0.42 & 0.11 & 1.13 & 4.44 & 3.93 \\
\hline $\mathbf{U}$ & 3832 & 4698 & 0.48 & 0.42 & 0.11 & 1.13 & 4.48 & 3.97 \\
\hline $\mathbf{V}$ & 3052 & 4909 & 0.47 & 0.42 & 0.11 & 1.13 & 4.46 & 3.97 \\
\hline W & 4185 & 4987 & 0.48 & 0.42 & 0.10 & 1.13 & 4.63 & 4.08 \\
\hline $\mathbf{X}$ & 3832 & 4999 & 0.48 & 0.42 & 0.10 & 1.13 & 4.62 & 4.08 \\
\hline $\mathbf{Y}$ & 3186 & 5016 & 0.47 & 0.42 & 0.11 & 1.13 & 4.42 & 3.93 \\
\hline $\mathrm{Z}$ & 3837 & 5589 & 0.47 & 0.42 & 0.11 & 1.13 & 4.46 & 3.95 \\
\hline $\mathbf{A A}$ & 3580 & 6080 & 0.48 & 0.42 & 0.11 & 1.13 & 4.51 & 4.00 \\
\hline AB & 4168 & 6198 & 0.48 & 0.42 & 0.11 & 1.13 & 4.51 & 4.00 \\
\hline $\mathbf{A C}$ & 3522 & 6360 & 0.48 & 0.42 & 0.11 & 1.13 & 4.54 & 4.01 \\
\hline AD & 4124 & 6693 & 0.47 & 0.42 & 0.11 & 1.13 & 4.43 & 3.93 \\
\hline $\mathbf{A E}$ & 3946 & 8069 & 0.48 & 0.42 & 0.10 & 1.14 & 4.86 & 4.26 \\
\hline $\mathbf{A F}$ & 3425 & 8325 & 0.48 & 0.42 & 0.10 & 1.12 & 4.56 & 4.06 \\
\hline AG & 3423 & 8389 & 0.48 & 0.42 & 0.11 & 1.13 & 4.51 & 4.00 \\
\hline AH & 4061 & 8552 & 0.48 & 0.42 & 0.10 & 1.13 & 4.63 & 4.09 \\
\hline AI & 4249 & 8696 & 0.48 & 0.42 & 0.10 & 1.13 & 4.61 & 4.08 \\
\hline AJ & 3967 & 8838 & 0.48 & 0.42 & 0.10 & 1.13 & 4.58 & 4.06 \\
\hline AK & 3425 & 8979 & 0.48 & 0.42 & 0.11 & 1.13 & 4.50 & 3.99 \\
\hline $\mathbf{A L}$ & 3410 & 9084 & 0.48 & 0.42 & 0.10 & 1.13 & 4.66 & 4.12 \\
\hline $\mathbf{A M}$ & 3932 & 9084 & 0.48 & 0.42 & 0.11 & 1.13 & 4.53 & 4.01 \\
\hline AN & 4067 & 9160 & 0.47 & 0.42 & 0.11 & 1.12 & 4.38 & 3.90 \\
\hline AO & 4046 & 9205 & 0.48 & 0.42 & 0.11 & 1.13 & 4.53 & 4.00 \\
\hline AP & 3527 & 9896 & 0.47 & 0.42 & 0.11 & 1.13 & 4.46 & 3.97 \\
\hline AQ & 4096 & 10082 & 0.48 & 0.42 & 0.11 & 1.13 & 4.51 & 4.00 \\
\hline $\mathbf{A R}$ & 3619 & 10328 & 0.48 & 0.42 & 0.11 & 1.13 & 4.53 & 4.01 \\
\hline AS & 3993 & 10339 & 0.48 & 0.42 & 0.10 & 1.13 & 4.65 & 4.11 \\
\hline AT & 3984 & 10477 & 0.47 & 0.42 & 0.11 & 1.13 & 4.48 & 3.98 \\
\hline $\mathbf{A U}$ & 4319 & 10684 & 0.48 & 0.42 & 0.10 & 1.13 & 4.60 & 4.06 \\
\hline AV & 3729 & 10719 & 0.48 & 0.42 & 0.11 & 1.13 & 4.55 & 4.03 \\
\hline AW & 4072 & 10722 & 0.47 & 0.42 & 0.11 & 1.12 & 4.41 & 3.92 \\
\hline $\mathbf{A X}$ & 3436 & 10768 & 0.47 & 0.42 & 0.11 & 1.13 & 4.48 & 3.97 \\
\hline AY & 4174 & 10981 & 0.47 & 0.42 & 0.11 & 1.13 & 4.33 & 3.84 \\
\hline $\mathbf{A Z}$ & 3505 & 11179 & 0.48 & 0.42 & 0.10 & 1.14 & 4.61 & 4.06 \\
\hline
\end{tabular}


MATEC Web of Conferences

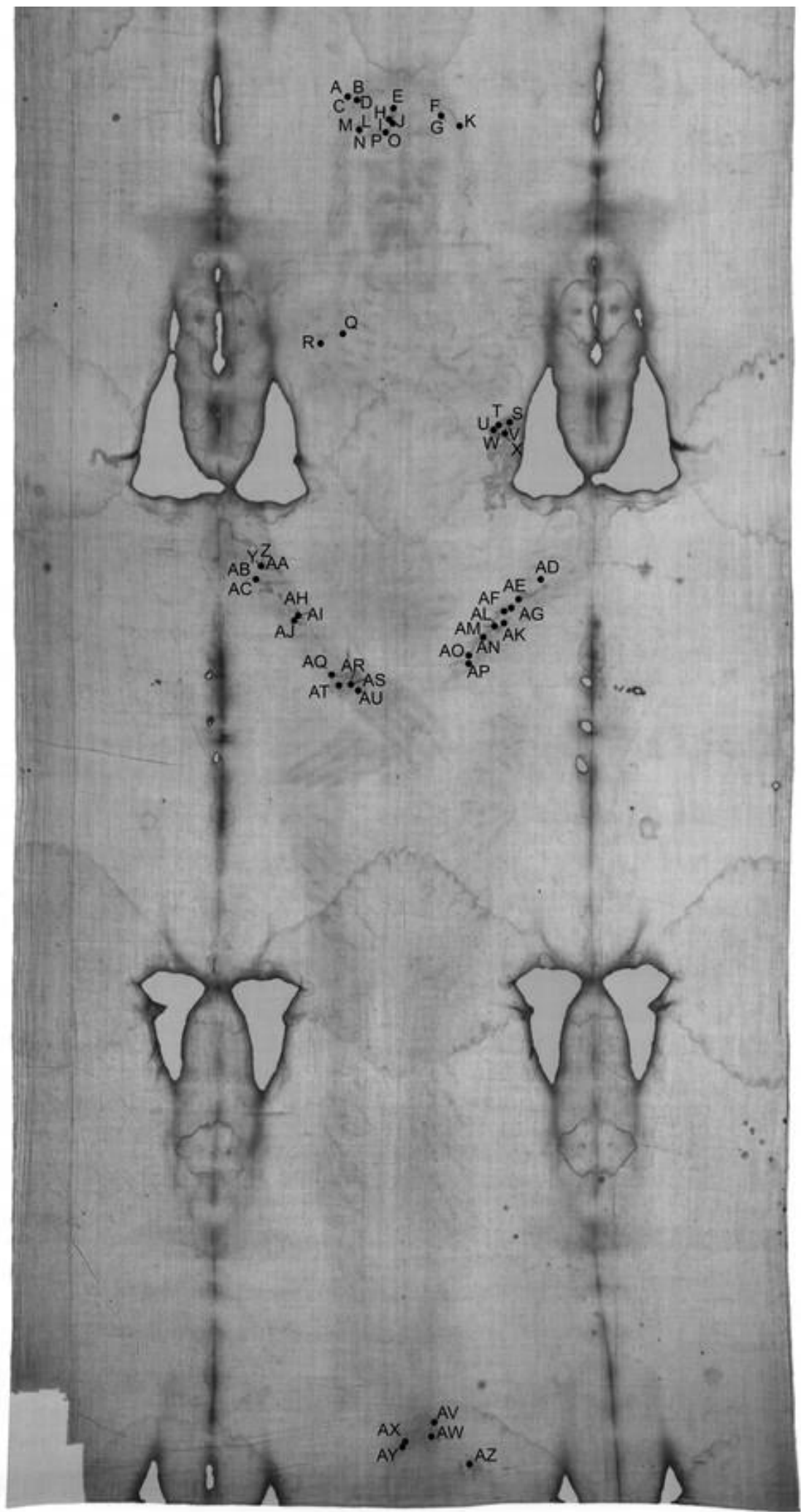

Figure 8. Measurement points on BLOOD front. 
Table 3. Reference table of the position in the (h, k) coordinate system of the measurement points in fig. 8 and related color values and dimensionless ratios.

\begin{tabular}{|c|c|c|c|c|c|c|c|c|}
\hline \multicolumn{9}{|c|}{ BLOOD chromatic values FRONT } \\
\hline & h & $\mathbf{k}$ & $x$ & $y$ & $z$ & $x / y$ & $x / z$ & $y / z$ \\
\hline $\mathbf{A}$ & 2877 & 662 & 0.49 & 0.42 & 0.10 & 1.18 & 5.15 & 4.38 \\
\hline B & 2954 & 683 & 0.49 & 0.42 & 0.09 & 1.18 & 5.20 & 4.43 \\
\hline C & 2947 & 696 & 0.50 & 0.42 & 0.09 & 1.19 & 5.55 & 4.67 \\
\hline D & 2953 & 696 & 0.50 & 0.42 & 0.09 & 1.19 & 5.49 & 4.62 \\
\hline $\mathbf{E}$ & 3222 & 746 & 0.49 & 0.42 & 0.10 & 1.17 & 5.08 & 4.35 \\
\hline $\mathbf{F}$ & 3564 & 803 & 0.49 & 0.42 & 0.10 & 1.17 & 5.17 & 4.41 \\
\hline $\mathbf{G}$ & 3591 & 818 & 0.49 & 0.42 & 0.09 & 1.18 & 5.24 & 4.43 \\
\hline $\mathbf{H}$ & 3195 & 846 & 0.49 & 0.42 & 0.09 & 1.18 & 5.44 & 4.61 \\
\hline I & 3209 & 860 & 0.49 & 0.41 & 0.10 & 1.19 & 5.07 & 4.28 \\
\hline $\mathbf{J}$ & 3222 & 869 & 0.49 & 0.42 & 0.09 & 1.18 & 5.39 & 4.58 \\
\hline $\mathbf{K}$ & 3718 & 883 & 0.49 & 0.42 & 0.09 & 1.17 & 5.26 & 4.51 \\
\hline $\mathbf{L}$ & 2975 & 900 & 0.49 & 0.42 & 0.09 & 1.18 & 5.31 & 4.52 \\
\hline $\mathbf{M}$ & 2950 & 906 & 0.49 & 0.42 & 0.09 & 1.17 & 5.21 & 4.44 \\
\hline $\mathbf{N}$ & 2980 & 924 & 0.49 & 0.42 & 0.09 & 1.18 & 5.29 & 4.50 \\
\hline O & 3173 & 928 & 0.49 & 0.42 & 0.09 & 1.18 & 5.43 & 4.61 \\
\hline $\mathbf{P}$ & 3163 & 945 & 0.49 & 0.42 & 0.09 & 1.18 & 5.51 & 4.68 \\
\hline $\mathbf{Q}$ & 2839 & 2436 & 0.49 & 0.42 & 0.09 & 1.17 & 5.56 & 4.77 \\
\hline $\mathbf{R}$ & 2671 & 2505 & 0.49 & 0.42 & 0.10 & 1.16 & 5.09 & 4.41 \\
\hline $\mathbf{S}$ & 4092 & 3099 & 0.49 & 0.42 & 0.10 & 1.17 & 5.02 & 4.29 \\
\hline $\mathbf{T}$ & 4002 & 3107 & 0.49 & 0.42 & 0.10 & 1.17 & 4.92 & 4.19 \\
\hline $\mathbf{U}$ & 3970 & 3155 & 0.49 & 0.42 & 0.10 & 1.17 & 4.97 & 4.23 \\
\hline $\mathbf{V}$ & 4045 & 3162 & 0.49 & 0.42 & 0.10 & 1.17 & 5.15 & 4.40 \\
\hline $\mathbf{W}$ & 4045 & 3169 & 0.49 & 0.42 & 0.10 & 1.17 & 5.17 & 4.41 \\
\hline $\mathbf{X}$ & 4054 & 3197 & 0.49 & 0.42 & 0.10 & 1.17 & 5.07 & 4.32 \\
\hline $\mathbf{Y}$ & 2226 & 4161 & 0.49 & 0.42 & 0.10 & 1.17 & 5.14 & 4.38 \\
\hline$Z$ & 2230 & 4167 & 0.49 & 0.42 & 0.10 & 1.17 & 5.08 & 4.33 \\
\hline $\mathbf{A A}$ & 2230 & 4168 & 0.49 & 0.42 & 0.10 & 1.17 & 5.01 & 4.28 \\
\hline $\mathbf{A B}$ & 2227 & 4179 & 0.48 & 0.42 & 0.10 & 1.16 & 4.79 & 4.13 \\
\hline $\mathbf{A C}$ & 2189 & 4267 & 0.48 & 0.42 & 0.10 & 1.16 & 4.85 & 4.17 \\
\hline AD & 4332 & 4270 & 0.49 & 0.42 & 0.09 & 1.17 & 5.53 & 4.71 \\
\hline $\mathbf{A E}$ & 4156 & 4411 & 0.49 & 0.42 & 0.10 & 1.17 & 5.04 & 4.33 \\
\hline $\mathbf{A F}$ & 4091 & 4478 & 0.48 & 0.42 & 0.10 & 1.16 & 4.91 & 4.24 \\
\hline AG & 4043 & 4510 & 0.49 & 0.42 & 0.10 & 1.17 & 5.02 & 4.29 \\
\hline $\mathbf{A H}$ & 2514 & 4539 & 0.49 & 0.41 & 0.10 & 1.18 & 4.88 & 4.14 \\
\hline AI & 2515 & 4539 & 0.49 & 0.42 & 0.10 & 1.17 & 4.86 & 4.14 \\
\hline $\mathbf{A J}$ & 2485 & 4572 & 0.49 & 0.42 & 0.10 & 1.17 & 4.87 & 4.19 \\
\hline AK & 4042 & 4598 & 0.49 & 0.42 & 0.09 & 1.18 & 5.44 & 4.63 \\
\hline $\mathbf{A L}$ & 3975 & 4613 & 0.49 & 0.42 & 0.10 & 1.16 & 4.97 & 4.27 \\
\hline $\mathbf{A M}$ & 3966 & 4614 & 0.49 & 0.42 & 0.10 & 1.16 & 4.89 & 4.21 \\
\hline AN & 3886 & 4701 & 0.49 & 0.42 & 0.10 & 1.17 & 5.08 & 4.36 \\
\hline AO & 3781 & 4835 & 0.48 & 0.42 & 0.10 & 1.16 & 4.85 & 4.18 \\
\hline AP & 3780 & 4895 & 0.49 & 0.42 & 0.09 & 1.16 & 5.19 & 4.46 \\
\hline AQ & 2751 & 4972 & 0.49 & 0.42 & 0.10 & 1.18 & 5.07 & 4.29 \\
\hline $\mathbf{A R}$ & 2910 & 5045 & 0.49 & 0.42 & 0.10 & 1.17 & 5.07 & 4.34 \\
\hline AS & 2915 & 5049 & 0.49 & 0.42 & 0.10 & 1.16 & 4.99 & 4.29 \\
\hline AT & 2797 & 5052 & 0.49 & 0.42 & 0.10 & 1.18 & 5.12 & 4.34 \\
\hline $\mathbf{A U}$ & 2966 & 5101 & 0.48 & 0.42 & 0.10 & 1.16 & 4.81 & 4.14 \\
\hline AV & 3526 & 10546 & 0.49 & 0.42 & 0.10 & 1.17 & 4.96 & 4.24 \\
\hline AW & 3502 & 10655 & 0.49 & 0.42 & 0.10 & 1.17 & 4.82 & 4.14 \\
\hline $\mathbf{A X}$ & 3292 & 10707 & 0.48 & 0.42 & 0.10 & 1.16 & 4.82 & 4.14 \\
\hline AY & 3277 & 10727 & 0.48 & 0.42 & 0.10 & 1.17 & 4.77 & 4.09 \\
\hline $\mathbf{A Z}$ & 3799 & 10862 & 0.49 & 0.42 & 0.10 & 1.17 & 4.91 & 4.20 \\
\hline
\end{tabular}


MATEC Web of Conferences

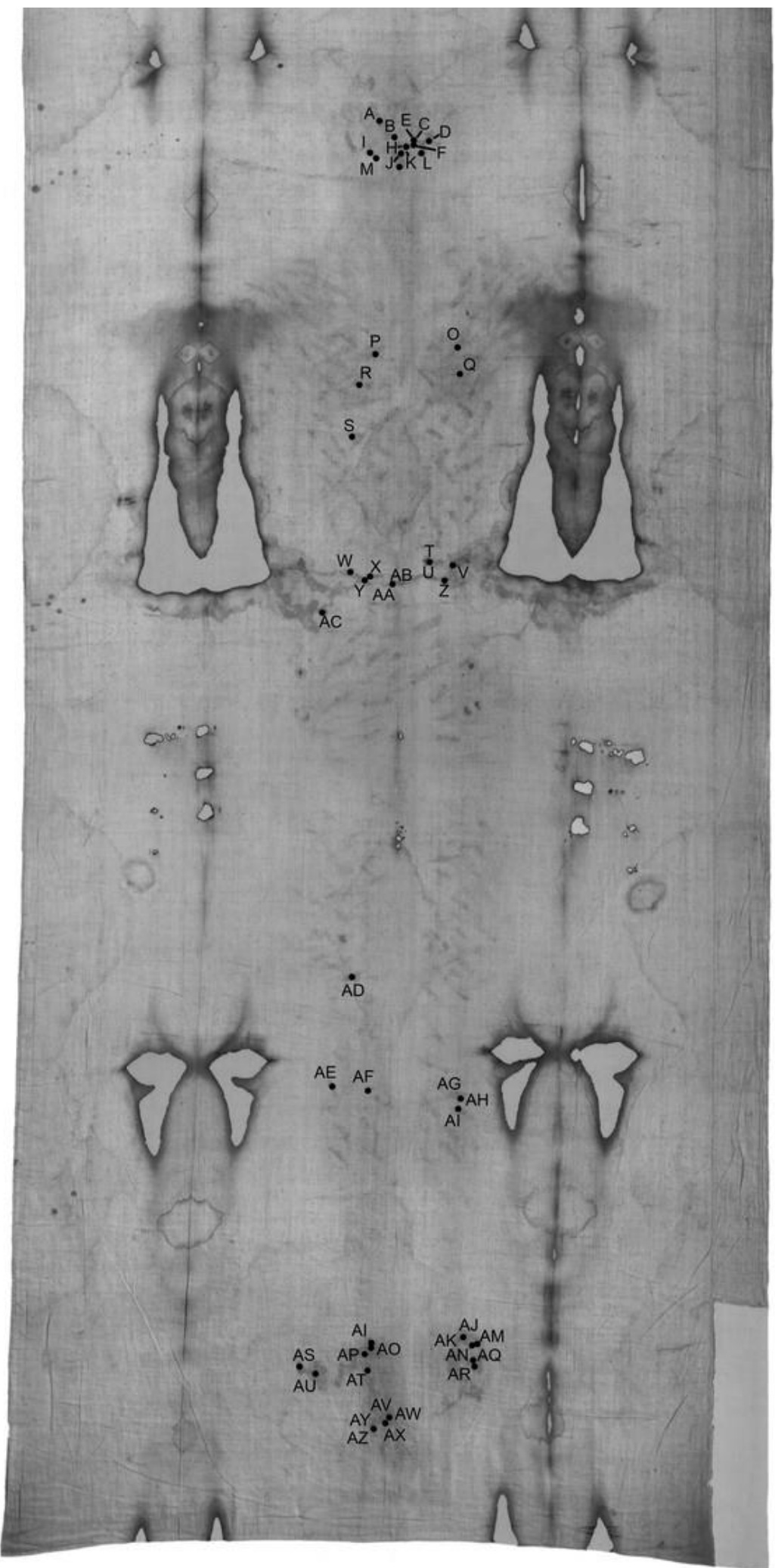

Figure 9. Measurement points on BLOOD back. 
Table 4. Reference table of the position in the (h, k) coordinate system of the measurement points in fig. 9 and related color values and dimensionless ratios.

\begin{tabular}{|c|c|c|c|c|c|c|c|c|}
\hline \multicolumn{9}{|c|}{ BLOOD chromatic values BACK } \\
\hline & $\mathbf{h}$ & $\mathbf{k}$ & $x$ & $y$ & $z$ & $x / y$ & $x / z$ & $y / z$ \\
\hline $\mathbf{A}$ & 3551 & 876 & 0.49 & 0.42 & 0.09 & 1.17 & 5.37 & 4.60 \\
\hline B & 3665 & 1003 & 0.50 & 0.42 & 0.09 & 1.19 & 5.60 & 4.72 \\
\hline $\mathbf{C}$ & 3821 & 1025 & 0.49 & 0.42 & 0.09 & 1.18 & 5.28 & 4.49 \\
\hline D & 3937 & 1033 & 0.49 & 0.42 & 0.09 & 1.18 & 5.21 & 4.43 \\
\hline $\mathbf{E}$ & 3819 & 1058 & 0.49 & 0.42 & 0.10 & 1.18 & 5.11 & 4.34 \\
\hline $\mathbf{F}$ & 3820 & 1058 & 0.49 & 0.42 & 0.10 & 1.18 & 5.09 & 4.32 \\
\hline $\mathbf{G}$ & 3762 & 1077 & 0.50 & 0.42 & 0.09 & 1.19 & 5.55 & 4.68 \\
\hline $\mathbf{H}$ & 3747 & 1077 & 0.49 & 0.42 & 0.09 & 1.18 & 5.25 & 4.46 \\
\hline I & 3476 & 1123 & 0.49 & 0.42 & 0.09 & 1.17 & 5.44 & 4.64 \\
\hline $\mathbf{J}$ & 3720 & 1127 & 0.49 & 0.41 & 0.10 & 1.18 & 4.88 & 4.14 \\
\hline K & 3727 & 1128 & 0.49 & 0.42 & 0.10 & 1.17 & 4.97 & 4.23 \\
\hline $\mathbf{L}$ & 3875 & 1131 & 0.49 & 0.42 & 0.09 & 1.18 & 5.31 & 4.49 \\
\hline M & 3522 & 1162 & 0.49 & 0.42 & 0.10 & 1.18 & 5.02 & 4.25 \\
\hline $\mathbf{N}$ & 3710 & 1232 & 0.49 & 0.42 & 0.10 & 1.17 & 5.16 & 4.40 \\
\hline O & 4155 & 2641 & 0.49 & 0.42 & 0.09 & 1.17 & 5.24 & 4.49 \\
\hline $\mathbf{P}$ & 3519 & 2693 & 0.50 & 0.42 & 0.09 & 1.19 & 5.60 & 4.73 \\
\hline $\mathbf{Q}$ & 4171 & 2848 & 0.49 & 0.42 & 0.09 & 1.18 & 5.47 & 4.65 \\
\hline $\mathbf{R}$ & 3395 & 2928 & 0.49 & 0.42 & 0.09 & 1.17 & 5.61 & 4.78 \\
\hline $\mathbf{S}$ & 3336 & 3337 & 0.49 & 0.42 & 0.09 & 1.17 & 5.70 & 4.88 \\
\hline $\mathbf{T}$ & 3940 & 4306 & 0.50 & 0.42 & 0.09 & 1.19 & 5.38 & 4.51 \\
\hline $\mathbf{U}$ & 3940 & 4311 & 0.49 & 0.42 & 0.09 & 1.19 & 5.41 & 4.55 \\
\hline V & 4118 & 4336 & 0.49 & 0.42 & 0.09 & 1.18 & 5.53 & 4.70 \\
\hline W & 3321 & 4386 & 0.49 & 0.42 & 0.09 & 1.17 & 5.39 & 4.62 \\
\hline$X$ & 3484 & 4419 & 0.49 & 0.42 & 0.09 & 1.17 & 5.44 & 4.66 \\
\hline $\mathbf{Y}$ & 3432 & 4450 & 0.49 & 0.42 & 0.10 & 1.16 & 4.89 & 4.21 \\
\hline $\mathbf{Z}$ & 4059 & 4453 & 0.49 & 0.42 & 0.09 & 1.17 & 5.25 & 4.47 \\
\hline $\mathbf{A A}$ & 3647 & 4481 & 0.49 & 0.42 & 0.09 & 1.17 & 5.18 & 4.42 \\
\hline $\mathbf{A B}$ & 3662 & 4486 & 0.49 & 0.42 & 0.09 & 1.18 & 5.53 & 4.70 \\
\hline AC & 3107 & 4701 & 0.49 & 0.42 & 0.09 & 1.17 & 5.28 & 4.52 \\
\hline AD & 3336 & 7537 & 0.49 & 0.42 & 0.09 & 1.17 & 5.58 & 4.76 \\
\hline $\mathbf{A E}$ & 3182 & 8386 & 0.49 & 0.42 & 0.09 & 1.16 & 5.19 & 4.49 \\
\hline $\mathbf{A F}$ & 3460 & 8427 & 0.49 & 0.42 & 0.10 & 1.15 & 5.03 & 4.36 \\
\hline AG & 4176 & 8484 & 0.49 & 0.42 & 0.10 & 1.16 & 5.00 & 4.32 \\
\hline AH & 4187 & 8486 & 0.49 & 0.42 & 0.09 & 1.16 & 5.25 & 4.53 \\
\hline AI & 4163 & 8563 & 0.49 & 0.42 & 0.09 & 1.16 & 5.53 & 4.75 \\
\hline AJ & 4207 & 10337 & 0.49 & 0.42 & 0.10 & 1.17 & 5.01 & 4.30 \\
\hline AK & 4191 & 10345 & 0.49 & 0.42 & 0.10 & 1.16 & 4.97 & 4.29 \\
\hline $\mathbf{A L}$ & 3487 & 10391 & 0.49 & 0.42 & 0.10 & 1.17 & 5.08 & 4.34 \\
\hline $\mathbf{A M}$ & 4309 & 10391 & 0.49 & 0.42 & 0.10 & 1.17 & 5.10 & 4.36 \\
\hline $\mathbf{A N}$ & 4272 & 10402 & 0.49 & 0.42 & 0.10 & 1.17 & 5.00 & 4.27 \\
\hline AO & 3487 & 10421 & 0.49 & 0.42 & 0.09 & 1.18 & 5.23 & 4.44 \\
\hline AP & 3434 & 10469 & 0.49 & 0.42 & 0.10 & 1.17 & 4.88 & 4.17 \\
\hline AQ & 4275 & 10523 & 0.49 & 0.42 & 0.10 & 1.17 & 4.97 & 4.27 \\
\hline $\mathbf{A R}$ & 4297 & 10567 & 0.48 & 0.42 & 0.10 & 1.16 & 4.88 & 4.20 \\
\hline AS & 2926 & 10570 & 0.49 & 0.42 & 0.09 & 1.18 & 5.44 & 4.60 \\
\hline AT & 3459 & 10600 & 0.49 & 0.42 & 0.10 & 1.17 & 4.91 & 4.22 \\
\hline $\mathbf{A U}$ & 3052 & 10625 & 0.49 & 0.42 & 0.09 & 1.17 & 5.19 & 4.42 \\
\hline AV & 3620 & 10965 & 0.49 & 0.41 & 0.10 & 1.19 & 5.10 & 4.30 \\
\hline $\mathbf{A W}$ & 3623 & 10971 & 0.49 & 0.42 & 0.09 & 1.18 & 5.21 & 4.41 \\
\hline $\mathbf{A X}$ & 3595 & 11009 & 0.49 & 0.42 & 0.10 & 1.17 & 4.92 & 4.23 \\
\hline AY & 3508 & 11048 & 0.49 & 0.42 & 0.10 & 1.17 & 5.07 & 4.33 \\
\hline $\mathbf{A Z}$ & 3506 & 11055 & 0.49 & 0.42 & 0.10 & 1.17 & 4.94 & 4.23 \\
\hline
\end{tabular}


MATEC Web of Conferences

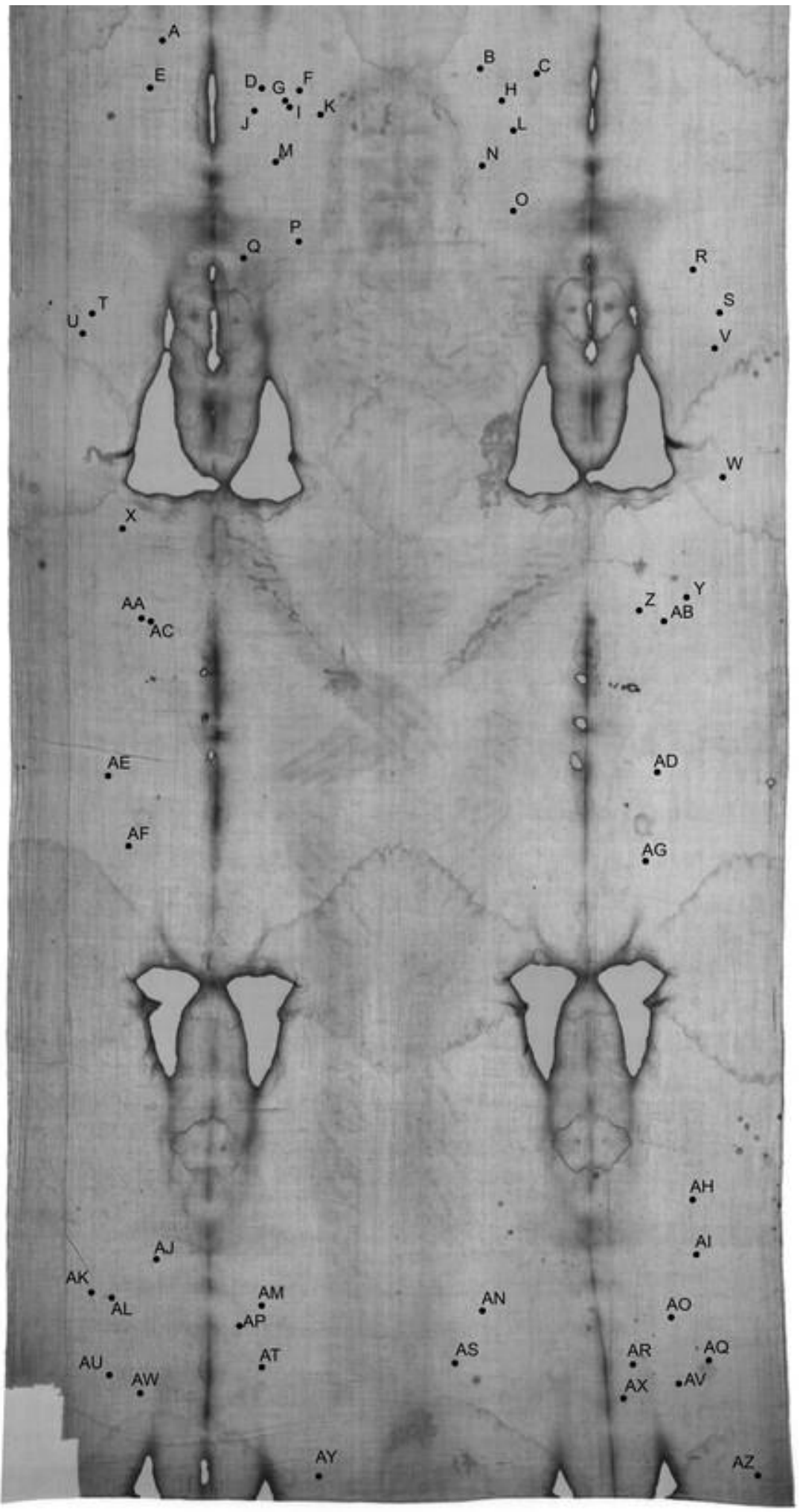

Figure 10. Measurement points on BACKGROUND front. 
Table 5. Reference table of the position in the (h, k) coordinate system of the measurement points in fig. 10 and related color values and dimensionless ratios.

\begin{tabular}{|c|c|c|c|c|c|c|c|c|}
\hline \multicolumn{9}{|c|}{ BACKGROUND chromatic values FRONT } \\
\hline & $\mathbf{h}$ & $\mathbf{k}$ & $x$ & $y$ & $z$ & $x / y$ & $x / z$ & $y / z$ \\
\hline $\mathbf{A}$ & 1539 & 257 & 0.47 & 0.42 & 0.11 & 1.13 & 4.21 & 3.74 \\
\hline B & 3910 & 466 & 0.47 & 0.42 & 0.11 & 1.12 & 4.17 & 3.73 \\
\hline C & 4329 & 496 & 0.47 & 0.42 & 0.11 & 1.12 & 4.30 & 3.83 \\
\hline D & 2293 & 600 & 0.47 & 0.42 & 0.11 & 1.12 & 4.11 & 3.66 \\
\hline $\mathbf{E}$ & 1455 & 614 & 0.47 & 0.42 & 0.11 & 1.13 & 4.22 & 3.74 \\
\hline $\mathbf{F}$ & 2551 & 629 & 0.47 & 0.42 & 0.11 & 1.13 & 4.37 & 3.88 \\
\hline $\mathbf{G}$ & 2452 & 692 & 0.47 & 0.42 & 0.11 & 1.12 & 4.13 & 3.68 \\
\hline $\mathbf{H}$ & 4068 & 706 & 0.47 & 0.42 & 0.11 & 1.12 & 4.22 & 3.77 \\
\hline I & 2479 & 757 & 0.47 & 0.42 & 0.11 & 1.12 & 4.16 & 3.71 \\
\hline $\mathbf{J}$ & 2218 & 789 & 0.47 & 0.42 & 0.11 & 1.12 & 4.18 & 3.72 \\
\hline $\mathbf{K}$ & 2705 & 810 & 0.47 & 0.42 & 0.11 & 1.13 & 4.31 & 3.83 \\
\hline $\mathbf{L}$ & 4157 & 924 & 0.47 & 0.42 & 0.11 & 1.12 & 4.27 & 3.82 \\
\hline M & 2379 & 1163 & 0.47 & 0.42 & 0.11 & 1.12 & 4.27 & 3.80 \\
\hline $\mathbf{N}$ & 3927 & 1190 & 0.47 & 0.42 & 0.11 & 1.12 & 4.23 & 3.78 \\
\hline O & 4147 & 1535 & 0.47 & 0.42 & 0.11 & 1.13 & 4.40 & 3.91 \\
\hline $\mathbf{P}$ & 2550 & 1763 & 0.48 & 0.42 & 0.11 & 1.13 & 4.45 & 3.94 \\
\hline $\mathbf{Q}$ & 2142 & 1874 & 0.47 & 0.42 & 0.11 & 1.13 & 4.40 & 3.91 \\
\hline $\mathbf{R}$ & 5448 & 1967 & 0.47 & 0.42 & 0.11 & 1.13 & 4.44 & 3.93 \\
\hline $\mathbf{S}$ & 5685 & 2295 & 0.47 & 0.42 & 0.11 & 1.12 & 4.27 & 3.81 \\
\hline $\mathbf{T}$ & 1006 & 2298 & 0.47 & 0.42 & 0.11 & 1.12 & 4.14 & 3.69 \\
\hline $\mathbf{U}$ & 933 & 2449 & 0.47 & 0.42 & 0.11 & 1.12 & 4.26 & 3.79 \\
\hline $\mathbf{V}$ & 5641 & 2557 & 0.47 & 0.42 & 0.11 & 1.13 & 4.46 & 3.96 \\
\hline W & 5720 & 3520 & 0.47 & 0.42 & 0.11 & 1.12 & 4.18 & 3.73 \\
\hline $\mathbf{X}$ & 1239 & 3906 & 0.47 & 0.42 & 0.11 & 1.13 & 4.28 & 3.81 \\
\hline Y & 5441 & 4413 & 0.47 & 0.42 & 0.11 & 1.12 & 4.40 & 3.91 \\
\hline $\mathbf{Z}$ & 5093 & 4517 & 0.47 & 0.42 & 0.11 & 1.12 & 4.23 & 3.78 \\
\hline $\mathbf{A A}$ & 1378 & 4582 & 0.47 & 0.42 & 0.11 & 1.12 & 4.23 & 3.77 \\
\hline $\mathbf{A B}$ & 5284 & 4600 & 0.47 & 0.42 & 0.11 & 1.12 & 4.21 & 3.77 \\
\hline $\mathbf{A C}$ & 1438 & 4603 & 0.47 & 0.42 & 0.11 & 1.12 & 4.10 & 3.65 \\
\hline AD & 5231 & 5726 & 0.47 & 0.42 & 0.11 & 1.12 & 4.22 & 3.76 \\
\hline $\mathbf{A E}$ & 1127 & 5757 & 0.47 & 0.42 & 0.11 & 1.13 & 4.43 & 3.93 \\
\hline $\mathbf{A F}$ & 1282 & 6277 & 0.48 & 0.42 & 0.11 & 1.13 & 4.47 & 3.95 \\
\hline AG & 5138 & 6398 & 0.47 & 0.42 & 0.11 & 1.12 & 4.37 & 3.90 \\
\hline $\mathbf{A H}$ & 5480 & 8927 & 0.48 & 0.42 & 0.10 & 1.13 & 4.55 & 4.04 \\
\hline AI & 5515 & 9333 & 0.48 & 0.42 & 0.10 & 1.13 & 4.55 & 4.04 \\
\hline AJ & 1494 & 9373 & 0.47 & 0.42 & 0.11 & 1.12 & 4.25 & 3.80 \\
\hline AK & 1001 & 9621 & 0.47 & 0.42 & 0.11 & 1.13 & 4.39 & 3.89 \\
\hline AL & 1151 & 9667 & 0.47 & 0.42 & 0.11 & 1.13 & 4.42 & 3.92 \\
\hline $\mathbf{A M}$ & 2283 & 9721 & 0.47 & 0.42 & 0.11 & 1.13 & 4.35 & 3.86 \\
\hline AN & 3905 & 9755 & 0.47 & 0.42 & 0.11 & 1.12 & 4.25 & 3.79 \\
\hline AO & 5325 & 9801 & 0.47 & 0.42 & 0.11 & 1.12 & 4.42 & 3.94 \\
\hline AP & 2115 & 9875 & 0.48 & 0.42 & 0.11 & 1.13 & 4.53 & 4.01 \\
\hline $\mathbf{A Q}$ & 5613 & 10137 & 0.48 & 0.42 & 0.10 & 1.13 & 4.56 & 4.03 \\
\hline $\mathbf{A R}$ & 5048 & 10156 & 0.48 & 0.42 & 0.11 & 1.13 & 4.52 & 4.01 \\
\hline AS & 3725 & 10157 & 0.47 & 0.42 & 0.11 & 1.12 & 4.28 & 3.81 \\
\hline AT & 2280 & 10198 & 0.48 & 0.42 & 0.11 & 1.13 & 4.47 & 3.95 \\
\hline $\mathbf{A U}$ & 1130 & 10234 & 0.48 & 0.42 & 0.11 & 1.13 & 4.49 & 3.98 \\
\hline $\mathbf{A V}$ & 5391 & 10294 & 0.47 & 0.42 & 0.11 & 1.12 & 4.32 & 3.85 \\
\hline AW & 1374 & 10372 & 0.48 & 0.42 & 0.10 & 1.14 & 4.86 & 4.26 \\
\hline $\mathbf{A X}$ & 4971 & 10401 & 0.48 & 0.42 & 0.10 & 1.14 & 4.76 & 4.19 \\
\hline AY & 2699 & 10991 & 0.48 & 0.42 & 0.10 & 1.15 & 5.02 & 4.37 \\
\hline $\mathbf{A Z}$ & 5993 & 10991 & 0.47 & 0.42 & 0.11 & 1.12 & 4.26 & 3.80 \\
\hline
\end{tabular}


MATEC Web of Conferences

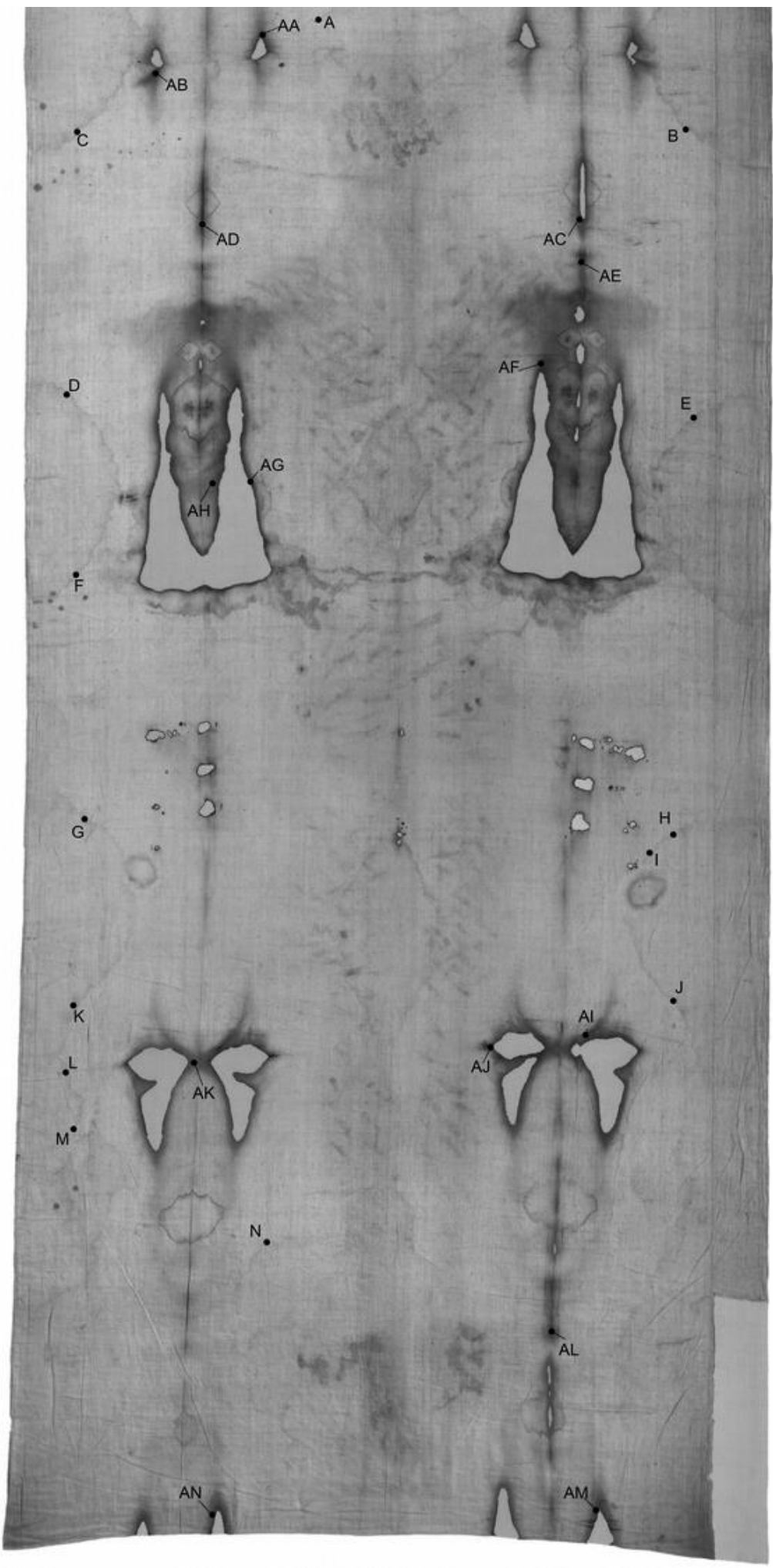

Figure 11. Measurement points on BACKGROUND back. 
Table 6. Reference table of the position in the (h, k) coordinate system of the measurement points in fig. 11 and related color values and dimensionless ratios.

\begin{tabular}{|c|c|c|c|c|c|c|c|c|}
\hline \multicolumn{9}{|c|}{ BACKGROUND chromatic values BACK } \\
\hline & $\mathbf{h}$ & $\mathbf{k}$ & $x$ & $y$ & $z$ & $x / y$ & $x / z$ & $y / z$ \\
\hline $\mathbf{A}$ & 4320 & 500 & 0.48 & 0.42 & 0.11 & 1.13 & 4.53 & 4.00 \\
\hline B & 2840 & 630 & 0.47 & 0.42 & 0.11 & 1.12 & 4.24 & 3.79 \\
\hline $\mathbf{C}$ & 2415 & 639 & 0.48 & 0.42 & 0.11 & 1.13 & 4.51 & 4.00 \\
\hline D & 2957 & 804 & 0.47 & 0.42 & 0.11 & 1.13 & 4.51 & 4.00 \\
\hline $\mathbf{E}$ & 4520 & 812 & 0.47 & 0.42 & 0.11 & 1.13 & 4.40 & 3.89 \\
\hline $\mathbf{F}$ & 2967 & 924 & 0.47 & 0.42 & 0.11 & 1.12 & 4.26 & 3.80 \\
\hline $\mathbf{G}$ & 4494 & 951 & 0.47 & 0.42 & 0.11 & 1.12 & 4.39 & 3.91 \\
\hline $\mathbf{H}$ & 4765 & 1111 & 0.47 & 0.42 & 0.11 & 1.13 & 4.33 & 3.84 \\
\hline I & 1263 & 1419 & 0.47 & 0.42 & 0.11 & 1.12 & 4.39 & 3.92 \\
\hline $\mathbf{J}$ & 1743 & 1441 & 0.47 & 0.42 & 0.11 & 1.12 & 4.21 & 3.76 \\
\hline $\mathbf{K}$ & 5582 & 1443 & 0.47 & 0.42 & 0.11 & 1.12 & 4.17 & 3.72 \\
\hline $\mathbf{L}$ & 5930 & 1954 & 0.47 & 0.42 & 0.11 & 1.12 & 4.15 & 3.70 \\
\hline M & 5759 & 2147 & 0.47 & 0.42 & 0.11 & 1.13 & 4.28 & 3.80 \\
\hline $\mathbf{N}$ & 1095 & 2174 & 0.48 & 0.42 & 0.10 & 1.13 & 4.55 & 4.04 \\
\hline O & 931 & 2395 & 0.48 & 0.42 & 0.10 & 1.13 & 4.57 & 4.04 \\
\hline $\mathbf{P}$ & 5961 & 2927 & 0.47 & 0.42 & 0.11 & 1.13 & 4.44 & 3.94 \\
\hline $\mathbf{Q}$ & 1209 & 2989 & 0.47 & 0.42 & 0.11 & 1.12 & 4.34 & 3.86 \\
\hline $\mathbf{R}$ & 1352 & 2996 & 0.47 & 0.42 & 0.11 & 1.12 & 4.10 & 3.66 \\
\hline $\mathbf{S}$ & 1566 & 3402 & 0.47 & 0.42 & 0.11 & 1.12 & 4.24 & 3.78 \\
\hline $\mathbf{T}$ & 1094 & 4650 & 0.47 & 0.42 & 0.11 & 1.12 & 4.29 & 3.82 \\
\hline $\mathbf{U}$ & 1600 & 4883 & 0.47 & 0.42 & 0.11 & 1.12 & 4.19 & 3.73 \\
\hline $\mathbf{V}$ & 5542 & 5079 & 0.47 & 0.42 & 0.11 & 1.13 & 4.24 & 3.77 \\
\hline W & 5316 & 5437 & 0.47 & 0.42 & 0.11 & 1.13 & 4.33 & 3.84 \\
\hline $\mathbf{X}$ & 5801 & 5572 & 0.47 & 0.42 & 0.11 & 1.13 & 4.38 & 3.88 \\
\hline $\mathbf{Y}$ & 1555 & 6257 & 0.47 & 0.42 & 0.12 & 1.12 & 4.08 & 3.64 \\
\hline $\mathrm{Z}$ & 5269 & 6490 & 0.47 & 0.42 & 0.11 & 1.12 & 4.15 & 3.71 \\
\hline $\mathbf{A A}$ & 1993 & 6586 & 0.47 & 0.42 & 0.11 & 1.12 & 4.22 & 3.77 \\
\hline AB & 4763 & 6738 & 0.47 & 0.42 & 0.11 & 1.12 & 4.22 & 3.76 \\
\hline $\mathbf{A C}$ & 1870 & 7112 & 0.47 & 0.42 & 0.11 & 1.12 & 4.29 & 3.83 \\
\hline AD & 4767 & 7134 & 0.47 & 0.42 & 0.11 & 1.12 & 4.15 & 3.70 \\
\hline $\mathbf{A E}$ & 5371 & 7221 & 0.47 & 0.42 & 0.11 & 1.12 & 4.12 & 3.68 \\
\hline $\mathbf{A F}$ & 1870 & 7518 & 0.47 & 0.42 & 0.11 & 1.12 & 4.18 & 3.73 \\
\hline AG & 5121 & 7574 & 0.47 & 0.42 & 0.12 & 1.12 & 4.05 & 3.61 \\
\hline AH & 2030 & 7594 & 0.47 & 0.42 & 0.11 & 1.12 & 4.29 & 3.82 \\
\hline AI & 1394 & 8765 & 0.47 & 0.42 & 0.11 & 1.13 & 4.28 & 3.80 \\
\hline $\mathbf{A J}$ & 2612 & 8885 & 0.47 & 0.42 & 0.11 & 1.12 & 4.23 & 3.77 \\
\hline AK & 2570 & 9441 & 0.47 & 0.42 & 0.11 & 1.12 & 4.25 & 3.79 \\
\hline $\mathbf{A L}$ & 5572 & 9645 & 0.47 & 0.42 & 0.11 & 1.12 & 4.15 & 3.69 \\
\hline $\mathbf{A M}$ & 5473 & 9774 & 0.47 & 0.42 & 0.11 & 1.12 & 4.11 & 3.67 \\
\hline AN & 5330 & 10381 & 0.47 & 0.42 & 0.11 & 1.13 & 4.25 & 3.77 \\
\hline AO & 5319 & 10559 & 0.47 & 0.42 & 0.11 & 1.13 & 4.22 & 3.75 \\
\hline AP & 1463 & 10703 & 0.47 & 0.42 & 0.11 & 1.12 & 4.24 & 3.78 \\
\hline AQ & 1607 & 10770 & 0.47 & 0.42 & 0.11 & 1.12 & 4.29 & 3.82 \\
\hline $\mathbf{A R}$ & 5684 & 10820 & 0.47 & 0.42 & 0.11 & 1.13 & 4.15 & 3.69 \\
\hline AS & 2520 & 11124 & 0.47 & 0.42 & 0.11 & 1.12 & 4.27 & 3.81 \\
\hline AT & 5132 & 11127 & 0.47 & 0.42 & 0.11 & 1.13 & 4.37 & 3.87 \\
\hline $\mathbf{A U}$ & 2764 & 11361 & 0.47 & 0.42 & 0.11 & 1.12 & 4.24 & 3.78 \\
\hline AV & 2399 & 11395 & 0.47 & 0.42 & 0.11 & 1.12 & 4.16 & 3.71 \\
\hline $\mathbf{A W}$ & 2608 & 11492 & 0.47 & 0.42 & 0.11 & 1.12 & 4.20 & 3.75 \\
\hline $\mathbf{A X}$ & 5572 & 11676 & 0.47 & 0.42 & 0.11 & 1.13 & 4.32 & 3.82 \\
\hline AY & 1085 & 11709 & 0.48 & 0.42 & 0.10 & 1.14 & 4.76 & 4.19 \\
\hline $\mathbf{A Z}$ & 4241 & 11752 & 0.47 & 0.42 & 0.11 & 1.13 & 4.21 & 3.74 \\
\hline
\end{tabular}


MATEC Web of Conferences

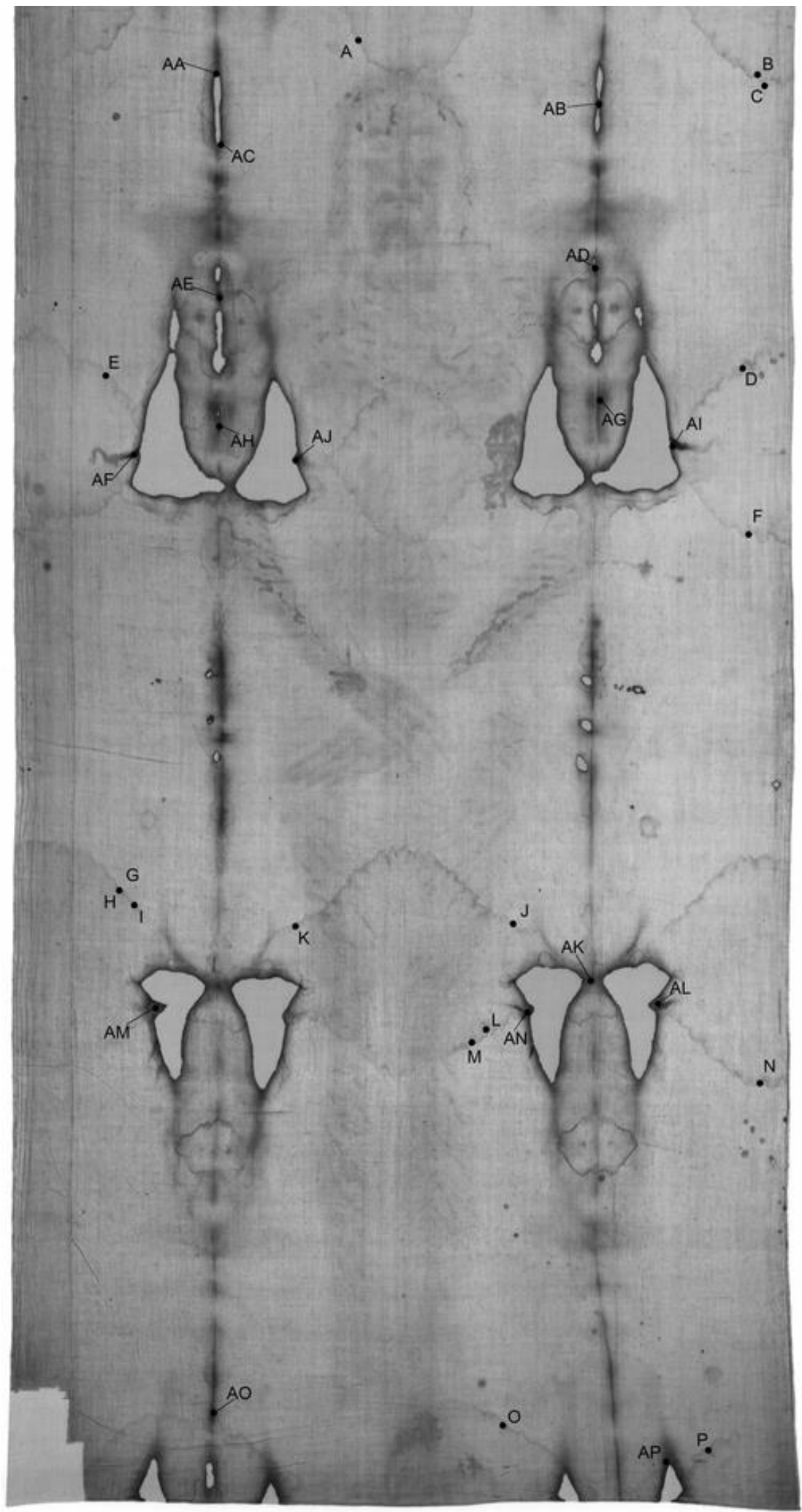

Figure 12. Measurement points on WATER STAINS and BURNS front. 
Table 7. Reference table of the position in the ( $h, k)$ coordinate system of the measurement points in fig. 12 and related color values and dimensionless ratios.

\begin{tabular}{|c|c|c|c|c|c|c|c|c|}
\hline \multicolumn{9}{|c|}{ WATER STAINS chromatic values FRONT } \\
\hline & h & $\mathbf{k}$ & $x$ & $y$ & $z$ & $x / y$ & $x / z$ & $y / z$ \\
\hline $\mathbf{A}$ & 2957 & 247 & 0.48 & 0.42 & 0.10 & 1.14 & 4.89 & 4.29 \\
\hline B & 5937 & 497 & 0.49 & 0.42 & 0.09 & 1.16 & 5.80 & 5.00 \\
\hline C & 5983 & 589 & 0.48 & 0.42 & 0.10 & 1.14 & 5.10 & 4.47 \\
\hline D & 5818 & 2698 & 0.49 & 0.42 & 0.08 & 1.16 & 5.89 & 5.07 \\
\hline $\mathbf{E}$ & 1073 & 2751 & 0.48 & 0.42 & 0.10 & 1.14 & 4.62 & 4.06 \\
\hline $\mathbf{F}$ & 5872 & 3930 & 0.48 & 0.42 & 0.10 & 1.14 & 4.99 & 4.37 \\
\hline $\mathbf{G}$ & 1176 & 6586 & 0.49 & 0.42 & 0.09 & 1.15 & 5.14 & 4.47 \\
\hline H & 1174 & 6589 & 0.49 & 0.42 & 0.10 & 1.15 & 5.13 & 4.46 \\
\hline I & 1289 & 6704 & 0.48 & 0.42 & 0.10 & 1.14 & 4.67 & 4.11 \\
\hline $\mathbf{J}$ & 4108 & 6840 & 0.48 & 0.42 & 0.10 & 1.14 & 5.00 & 4.39 \\
\hline $\mathbf{K}$ & 2486 & 6861 & 0.48 & 0.42 & 0.10 & 1.14 & 4.85 & 4.26 \\
\hline $\mathbf{L}$ & 3914 & 7625 & 0.48 & 0.42 & 0.10 & 1.14 & 5.06 & 4.45 \\
\hline M & 5957 & 8033 & 0.49 & 0.42 & 0.09 & 1.15 & 5.22 & 4.55 \\
\hline $\mathbf{N}$ & 4040 & 10583 & 0.48 & 0.42 & 0.10 & 1.14 & 4.84 & 4.26 \\
\hline O & 1112 & 10705 & 0.49 & 0.42 & 0.10 & 1.17 & 5.11 & 4.39 \\
\hline $\mathbf{P}$ & 5565 & 10768 & 0.49 & 0.42 & 0.09 & 1.16 & 5.36 & 4.63 \\
\hline \multicolumn{9}{|c|}{ BURNS chromatic values FRONT } \\
\hline & h & $\mathbf{k}$ & $\mathbf{x}$ & $\mathbf{y}$ & $\mathbf{z}$ & $x / y$ & $\mathbf{x} / \mathbf{z}$ & $y / z$ \\
\hline $\mathbf{A A}$ & 1895 & 494 & 0.50 & 0.41 & 0.09 & 1.22 & 5.34 & 4.40 \\
\hline $\mathbf{A B}$ & 4747 & 718 & 0.49 & 0.41 & 0.10 & 1.20 & 4.95 & 4.11 \\
\hline $\mathbf{A C}$ & 1931 & 1023 & 0.50 & 0.41 & 0.09 & 1.21 & 5.89 & 4.87 \\
\hline AD & 4718 & 1944 & 0.50 & 0.41 & 0.10 & 1.20 & 5.20 & 4.32 \\
\hline $\mathbf{A E}$ & 1922 & 2165 & 0.49 & 0.41 & 0.11 & 1.19 & 4.40 & 3.68 \\
\hline $\mathbf{A F}$ & 1277 & 2245 & 0.45 & 0.41 & 0.14 & 1.12 & 3.24 & 2.90 \\
\hline AG & 4760 & 2932 & 0.49 & 0.41 & 0.10 & 1.21 & 4.87 & 4.02 \\
\hline $\mathbf{A H}$ & 1912 & 3125 & 0.49 & 0.41 & 0.10 & 1.21 & 4.91 & 4.05 \\
\hline AI & 5319 & 3254 & 0.45 & 0.41 & 0.14 & 1.11 & 3.26 & 2.95 \\
\hline AJ & 2500 & 3369 & 0.47 & 0.40 & 0.13 & 1.18 & 3.73 & 3.16 \\
\hline $\mathbf{A K}$ & 4685 & 7260 & 0.50 & 0.41 & 0.10 & 1.22 & 5.06 & 4.16 \\
\hline $\mathbf{A L}$ & 5194 & 7434 & 0.50 & 0.42 & 0.08 & 1.17 & 5.92 & 5.05 \\
\hline AM & 1444 & 7469 & 0.50 & 0.42 & 0.09 & 1.20 & 5.57 & 4.65 \\
\hline AN & 4204 & 7515 & 0.48 & 0.41 & 0.11 & 1.20 & 4.31 & 3.61 \\
\hline AO & 1875 & 10497 & 0.48 & 0.41 & 0.11 & 1.18 & 4.50 & 3.81 \\
\hline AP & 5257 & 10854 & 0.47 & 0.40 & 0.13 & 1.16 & 3.71 & 3.19 \\
\hline
\end{tabular}


MATEC Web of Conferences

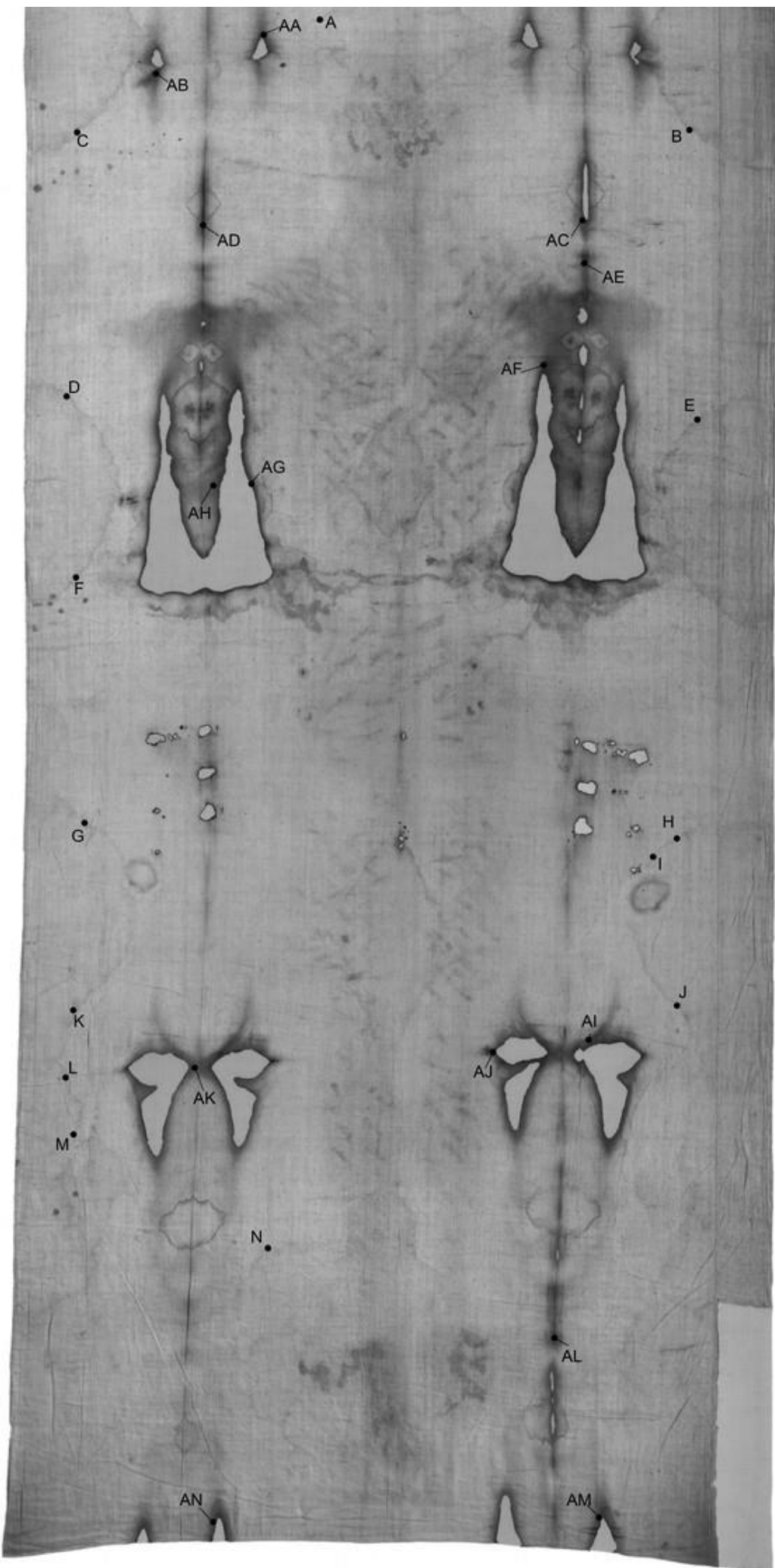

Figure 13. Measurement points on WATER STAINS and BURNS back. 
Table 8. Reference table of the position in the (h, k) coordinate system of the measurement points in fig. 13 and related color values and dimensionless ratios.

\begin{tabular}{|c|c|c|c|c|c|c|c|c|}
\hline \multicolumn{9}{|c|}{ WATER STAINS chromatic values BACK } \\
\hline & $\mathbf{h}$ & $\mathbf{k}$ & $x$ & $y$ & $z$ & $x / y$ & $x / z$ & $y / z$ \\
\hline $\mathbf{A}$ & 3082 & 93 & 0.48 & 0.42 & 0.10 & 1.14 & 4.94 & 4.35 \\
\hline B & 5951 & 953 & 0.48 & 0.42 & 0.10 & 1.14 & 4.85 & 4.26 \\
\hline $\mathrm{C}$ & 1196 & 970 & 0.48 & 0.42 & 0.10 & 1.14 & 5.02 & 4.41 \\
\hline D & 1119 & 3022 & 0.48 & 0.42 & 0.10 & 1.14 & 4.81 & 4.23 \\
\hline $\mathbf{E}$ & 6008 & 3204 & 0.49 & 0.42 & 0.10 & 1.15 & 5.13 & 4.46 \\
\hline $\mathbf{F}$ & 1188 & 4430 & 0.48 & 0.42 & 0.09 & 1.14 & 5.17 & 4.54 \\
\hline G & 1254 & 6339 & 0.48 & 0.42 & 0.10 & 1.14 & 4.70 & 4.13 \\
\hline H & 5850 & 6461 & 0.47 & 0.42 & 0.11 & 1.13 & 4.33 & 3.85 \\
\hline I & 5668 & 6604 & 0.48 & 0.42 & 0.11 & 1.13 & 4.42 & 3.90 \\
\hline $\mathbf{J}$ & 5854 & 7762 & 0.48 & 0.42 & 0.11 & 1.13 & 4.46 & 3.95 \\
\hline $\mathbf{K}$ & 1167 & 7796 & 0.48 & 0.42 & 0.10 & 1.14 & 5.07 & 4.44 \\
\hline $\mathbf{L}$ & 1107 & 8322 & 0.48 & 0.42 & 0.10 & 1.14 & 4.70 & 4.14 \\
\hline M & 1169 & 8760 & 0.48 & 0.42 & 0.09 & 1.14 & 5.19 & 4.54 \\
\hline $\mathbf{N}$ & 2678 & 9647 & 0.48 & 0.42 & 0.10 & 1.13 & 4.61 & 4.09 \\
\hline 0 & 3082 & 93 & 0.48 & 0.42 & 0.10 & 1.14 & 4.94 & 4.35 \\
\hline $\mathbf{P}$ & 5951 & 953 & 0.48 & 0.42 & 0.10 & 1.14 & 4.85 & 4.26 \\
\hline \multicolumn{9}{|c|}{ BURNS chromatic values BACK } \\
\hline & h & $\mathbf{k}$ & $\mathbf{x}$ & $\mathbf{y}$ & $\mathbf{Z}$ & $\mathbf{x} / \mathbf{y}$ & $\mathbf{x} / \mathbf{z}$ & $y / z$ \\
\hline $\mathbf{A A}$ & 2632 & 214 & 0.50 & 0.42 & 0.08 & 1.21 & 6.01 & 4.99 \\
\hline $\mathbf{A B}$ & 1797 & 514 & 0.50 & 0.41 & 0.09 & 1.20 & 5.32 & 4.42 \\
\hline $\mathbf{A C}$ & 5103 & 1653 & 0.49 & 0.41 & 0.09 & 1.20 & 5.29 & 4.43 \\
\hline $\mathbf{A D}$ & 2158 & 1695 & 0.49 & 0.41 & 0.10 & 1.20 & 4.91 & 4.09 \\
\hline $\mathbf{A E}$ & 5118 & 1989 & 0.50 & 0.42 & 0.09 & 1.20 & 5.82 & 4.85 \\
\hline AF & 4801 & 2779 & 0.50 & 0.41 & 0.10 & 1.20 & 5.22 & 4.34 \\
\hline $\mathbf{A G}$ & 2536 & 3704 & 0.49 & 0.41 & 0.11 & 1.19 & 4.61 & 3.87 \\
\hline $\mathbf{A H}$ & 2238 & 3716 & 0.50 & 0.42 & 0.08 & 1.20 & 6.20 & 5.17 \\
\hline AI & 5158 & 8019 & 0.50 & 0.41 & 0.09 & 1.21 & 5.38 & 4.43 \\
\hline AJ & 4406 & 8115 & 0.46 & 0.41 & 0.13 & 1.12 & 3.42 & 3.05 \\
\hline $\mathbf{A K}$ & 2098 & 8244 & 0.49 & 0.42 & 0.09 & 1.18 & 5.45 & 4.61 \\
\hline $\mathbf{A L}$ & 4890 & 10349 & 0.49 & 0.41 & 0.10 & 1.21 & 4.75 & 3.92 \\
\hline $\mathbf{A M}$ & 5234 & 11741 & 0.48 & 0.41 & 0.11 & 1.16 & 4.47 & 3.84 \\
\hline AN & 2236 & 11774 & 0.49 & 0.42 & 0.09 & 1.18 & 5.33 & 4.51 \\
\hline AO & 2632 & 214 & 0.50 & 0.42 & 0.08 & 1.21 & 6.01 & 4.99 \\
\hline $\mathbf{A P}$ & 1797 & 514 & 0.50 & 0.41 & 0.09 & 1.20 & 5.32 & 4.42 \\
\hline
\end{tabular}

Table 9. Dimensionless ratio range and averages of the analyzed features.

\begin{tabular}{|c|c|c|c|c|c|c|c|c|}
\hline & $\begin{array}{c}\boldsymbol{x} / \boldsymbol{y} \\
\min \end{array}$ & $\begin{array}{c}\boldsymbol{x} / \boldsymbol{y} \\
\max \end{array}$ & $\begin{array}{c}\boldsymbol{x} / \boldsymbol{z} \\
\min \end{array}$ & $\begin{array}{c}\boldsymbol{x} / \boldsymbol{z} \\
\max \end{array}$ & $\begin{array}{c}\boldsymbol{x} / \boldsymbol{y} \\
\text { average }\end{array}$ & $\begin{array}{c}\boldsymbol{x} / \boldsymbol{z} \\
\text { average }\end{array}$ & $\begin{array}{c}\boldsymbol{x} / \boldsymbol{y} \\
\min \end{array}$ & $\begin{array}{c}\boldsymbol{x} / \boldsymbol{y} \\
\max \end{array}$ \\
\hline Body image & 1.12 & 1.15 & 4.32 & 5.25 & 1.13 & 4.73 & 1.12 & 1.15 \\
\hline Background & 1.11 & 1.13 & 3.87 & 4.54 & 1.12 & 4.21 & 1.11 & 1.13 \\
\hline Blood & 1.15 & 1.19 & 4.77 & 5.70 & 1.17 & 5.16 & 1.15 & 1.19 \\
\hline Waterstains & 1.12 & 1.16 & 4.33 & 5.88 & 1.14 & 4.96 & 1.12 & 1.16 \\
\hline Burns & 1.10 & 1.21 & 3.23 & 6.20 & 1.18 & 4.90 & 1.10 & 1.21 \\
\hline
\end{tabular}




\subsection{Results on body image}

It can be seen from the plot in Figure 14 that the body image points, placed close to the area of blood, are characterized by $\mathrm{x} / \mathrm{z}$ values greater than 5 . Those points were found on the face and on the neck: the areas in question can be interpreted as typical of body image soaked with blood resulting from injuries on TS man's face and derived from the crown of thorns.

There are, as lower extreme, a series of feature points (ratio x/y goes from 1.12 to 1.13 , and ratio $\mathrm{x} / \mathrm{z}$ goes from 4.32 to 4.58 ) which overlap with the values area determined for the background: many of the image points that falling in the above mentioned value ranges, are located in the feet, the legs and lower back areas of the TS man.

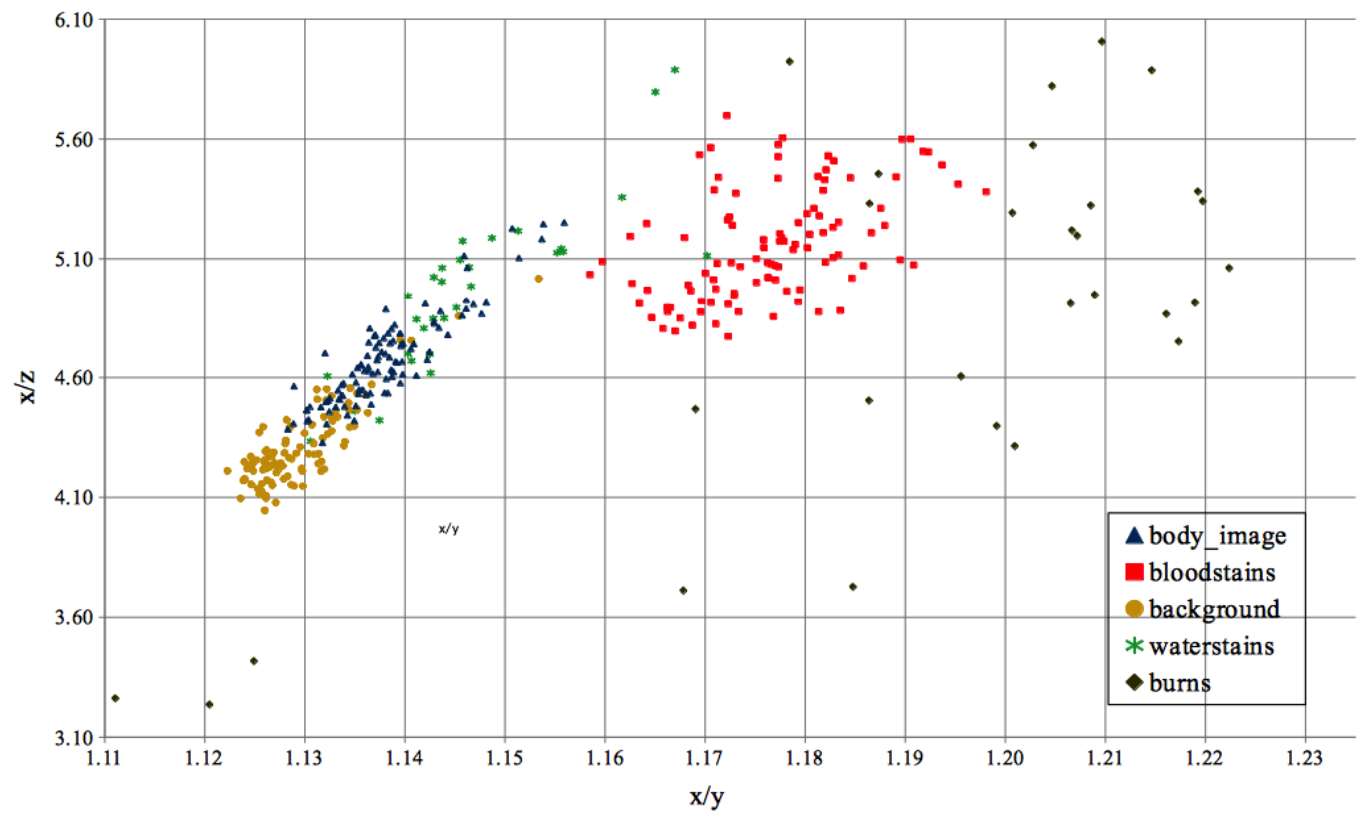

Figure 14. Plot of chromatic values ratio of all TS details.

\subsection{Results on body image}

It can be observed from the plot in Figure 14 that there is a not clear colorimetric separation between background and the body image: the color values change with continuity from the typical tone of the body image to those of the background, with a zone of interpenetration between the two "clouds" of data. This evidence is in agreement with some hypotheses of the body image formation that indicate it as the result of a premature aging of the linen caused probably by an radiative effect. Under this assumption, in fact, it is possible to hypothesize that the linen coloration occurred in a progressive manner, with varying intensity depending by the distance body-cloth.

The partial overlap of the values obtained for both, the image and the background, indicates a gradual color transition which extends along a narrow band of values, comparable for the two discussed features.

In order to verify a possible correlation between the color change and the distance from between cloth and body, some points close to the head of the TS man were taken where there is no apparently trace of the body image.

The typical values of these points have a values range for $\mathrm{x} / \mathrm{y}$ that goes from 1.12 to 1.13 with an arithmetical average of 1.12 , while for the $\mathrm{x} / \mathrm{z}$ ratio the values range goes from 4.25 to 4.52 with an arithmetical average of 4.39 . 
The author assumed that the chromatic variation can be related to a radiative effect (the same that could have originated to the body image), which has acted with variable intensity depending on the distance from the emission generation point.

\subsection{Results on blood stains.}

The "cloud" of values resulting from the color sampling on the blood appears positioned in the upper side of the plot used to represent the data (Fig. 14).

It is possible to identify the range of values within which scourge wounds are placed: the $x / y$ values ranging from 1.15 to 1.18 and the $\mathrm{x} / \mathrm{z}$ ranging from 4.99 to 5.69 .

In the lower part of the diagram (Fig. 15), characterized by $x / z$ values of less than 5 , the measurements results of the blood spilled by the feet and by the pulse of the Man of the Shroud can be seen.

Some color samplings have been performed on the chest's blood stain and on the visible blood stains outside of the body image, especially near to the left arm and near both feet on the front and back side of TS. A correlation between the blood of the chest and the one of the feet can be observed (Fig. 16), but the outside-arm blood seems different, this is also confirmed from visual examination of this feature.

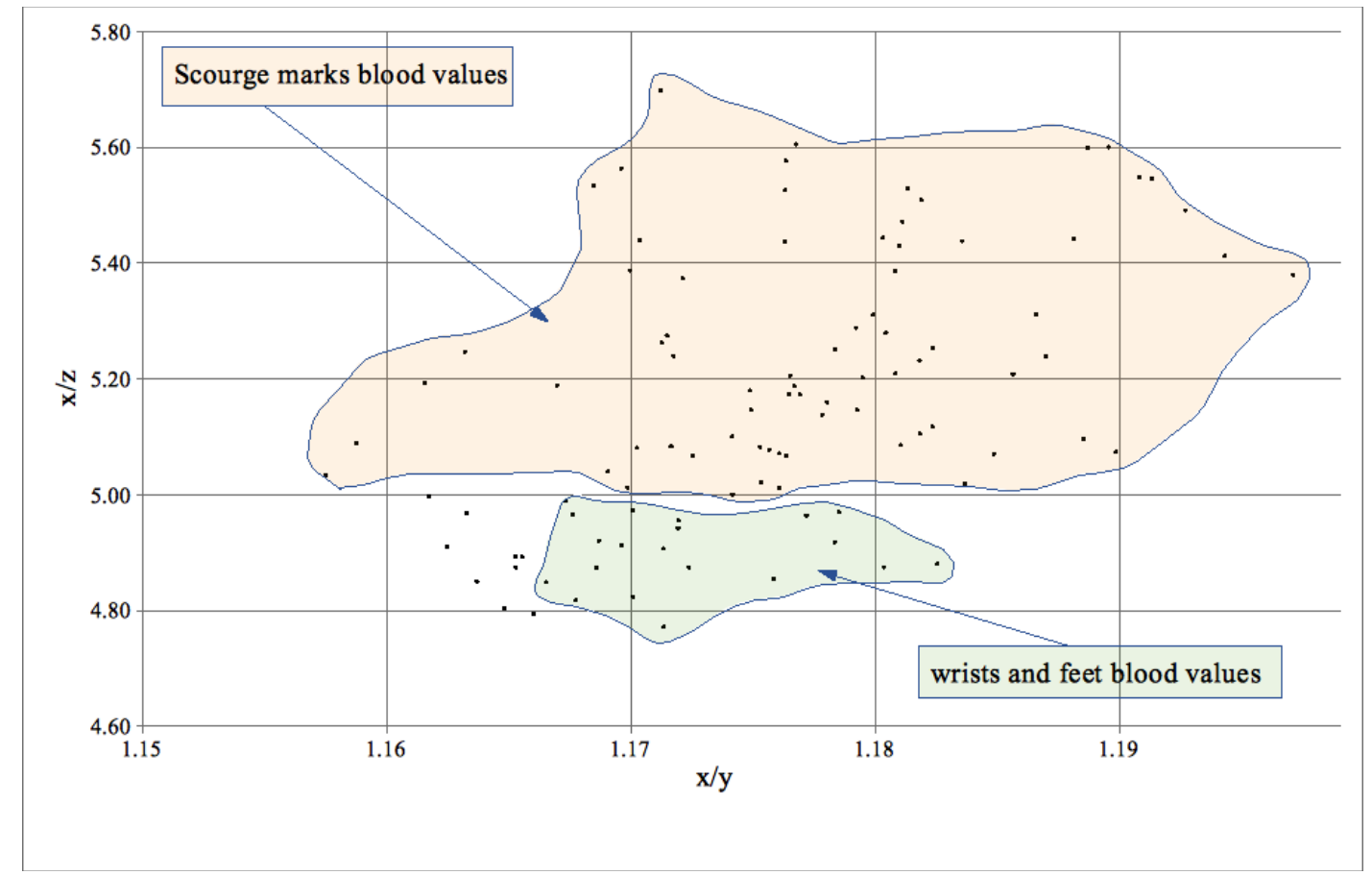

Figure 15. Plot of blood chromatic ratio values.

\subsection{Results on water stains.}

The color results of sampling on the edges of the tracks that are supposed to have been left by the water in a period prior to the Chambéry's fire, are mainly located in the area occupied by the values ratio results obtained for body image (Fig. 14), overlapping the same.

Analyzing the results (Fig, 17), it appears evident how the traces left by water (or maybe by another liquid) are comparable in colorimetric terms with the findings on the body image. 


\section{MATEC Web of Conferences}

\subsection{Results on burns}

The last series of color sampling, regards the colorimetric characterization of the burns left on the Shroud by the Chambéry's fire. It can be seen from the plots (Fig. 14 and 17) that the results of the dimensionless ratios place themselves mainly on the right side of the plots.

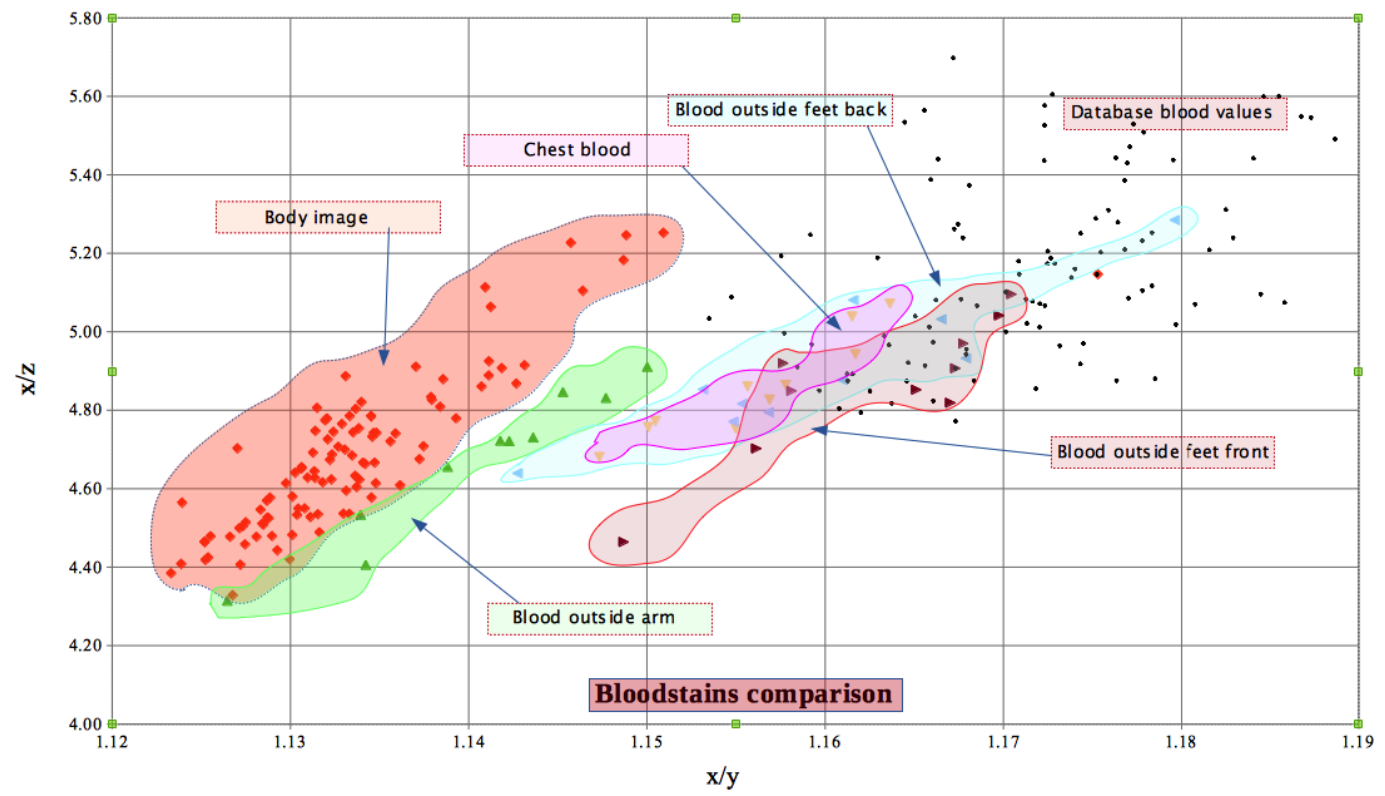

Figure 16. Blood stains comparison plot.

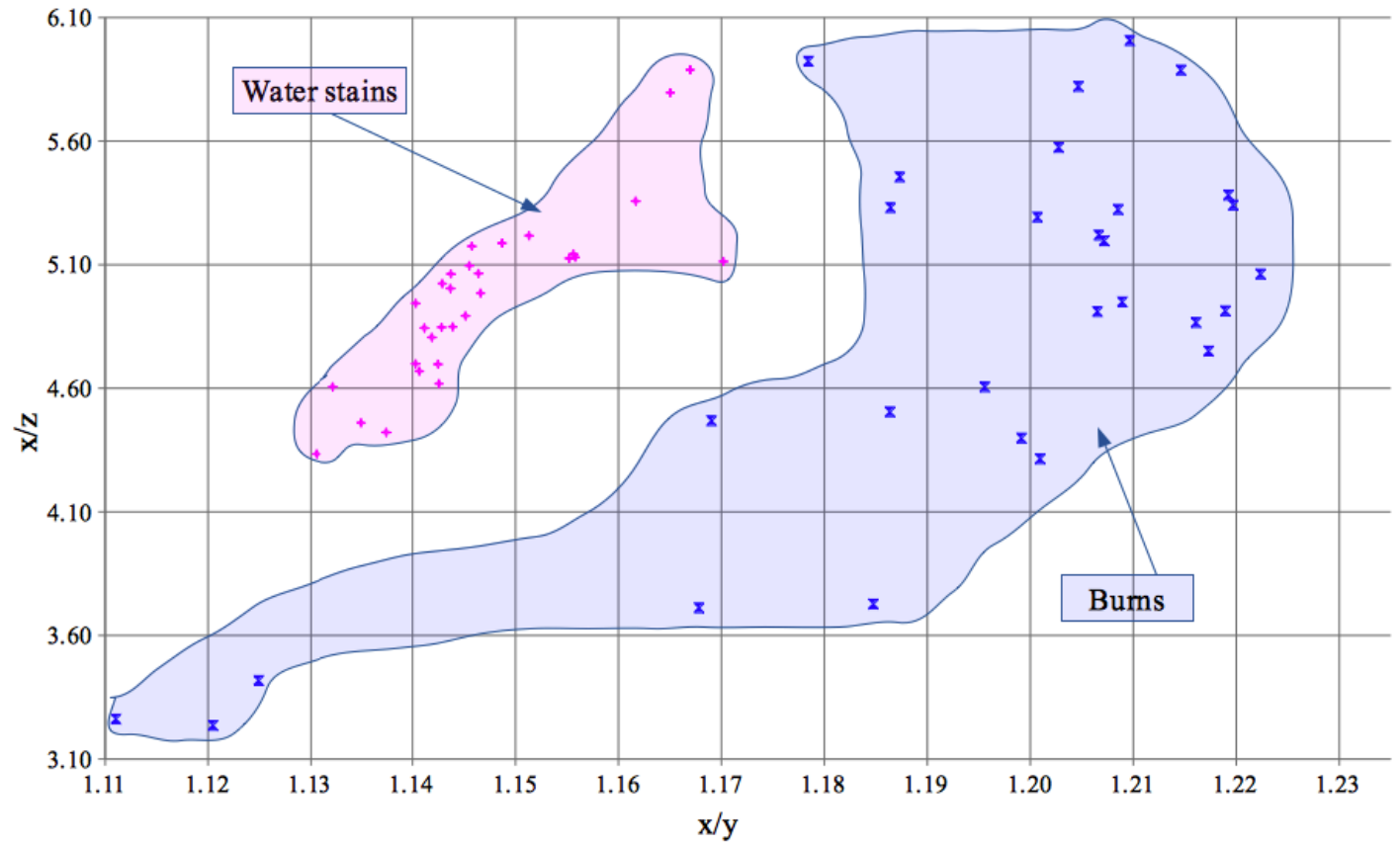

Figure 17. Plot of water stains and burns chromatic ratio values. 


\section{An application}

The created database has been useful for some study on TS features, performed by comparing new color samplings with the database measurements. Considering that the colorimetric values of TS's features are placed in "clouds", more than one measurement for the same TS detail is needed, because must to be evaluated the data trend.

So some study on symmetrical spots (Fig. 18) present on the glutes Man of the Shroud were conducted. These features, whose classification is still debated among researchers, appear located at the vertices of two triangles placed symmetrically to the axis of the back. This traces have blood stains appearance and some experts speculate that they may be traces of blood derived from the wounds generated by a pair of "tridents" on which TS man was forced to sit during the crucifixion.

By a visual inspection, these details appear surrounded by circular halos that can be traced to the serum which has been supposedly gathered around the wounds. For the author they appear too regular in shape and too extended to make possible to assimilate the traces left by blood serum.

A careful observation of this features points out that the central part of the spots appears so confused as to make the weave of the fabric not recognizable. This characteristic was not found in the details related to the blood stains. It may also be noticed that the tone of the central part goes from a very dark brown to a red-orange: also this feature has not been found on the blood stains. It can be assumed that the analyzed details were not originated by blood spilled from wounds.

In order to have more indicative elements, the analysis was carried out by performing more measurements on this circular stains. The results obtained on color value ratios can be observed in the plot reported in Figure 19 and they can be traced back to the typical values found for the burns.

The analysis of the evidence gathered and the assessments made tend to rule out that the currently analyzed details could be related to traces of blood, but rather to burns.

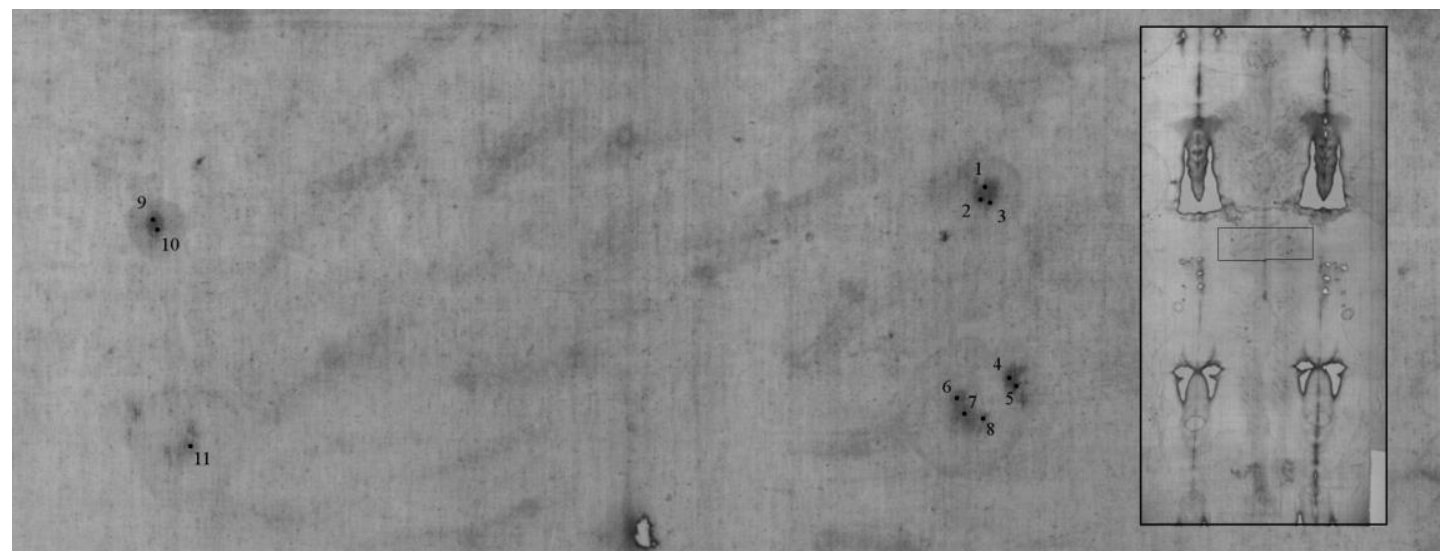

Figure 18. Position of analyzed unknown detail.

\section{Conclusions}

A colorimetric measurement process has been developed which allowed to obtain a TS calibrated quantitative image with $\pm 4 \%$ uncertainty relative to the XYZ color values. This result was achieved by comparison and correction of a digitalized photography made by G.Durante in 2002, with a previous published color analysis performed in CIE XYZ color space by P. Soardo et al. in 1978. The color samplings on digital image were made by using a common photographic software (Paint Shop ${ }^{\mathrm{TM}}$ Pro(C) with which the values of the single RGB color channel were read by means of the histogram function.

With the TS calibrated image, accurate color measurements were made possible and by properly interpreting the obtained results it was possible to analyze and classify some features. 


\section{MATEC Web of Conferences}

The color characteristics of body image, background, blood stains, water stain and burns were been investigated: the results give some information like the continuous color transition from background to image.

From a colorimetric point of view, the results on the blood stains have allowed to note that the area occupied by the blood is separated from that generated both from the background and from the body image. Differences between the color values of the blood derived from scourge wounds and the one flowed out from the deepest wounds were also identified. A feature on which future studies can be performed concerning the alleged blood outside the left arm: from colorimetric analysis it did not seems to have the typical colorimetric features found on other bloodstains.

The water stains, are ranked in the plot (Fig. 14) above those determined for the body image, extending also in a similar way: this result could suggest some possible correlation between body image and water stains.

The verification made by new colorimetric measurements on features, not analyzed during database construction, gave the expected results, also providing some additional information for example on visible details on the glutes of the TS Man.

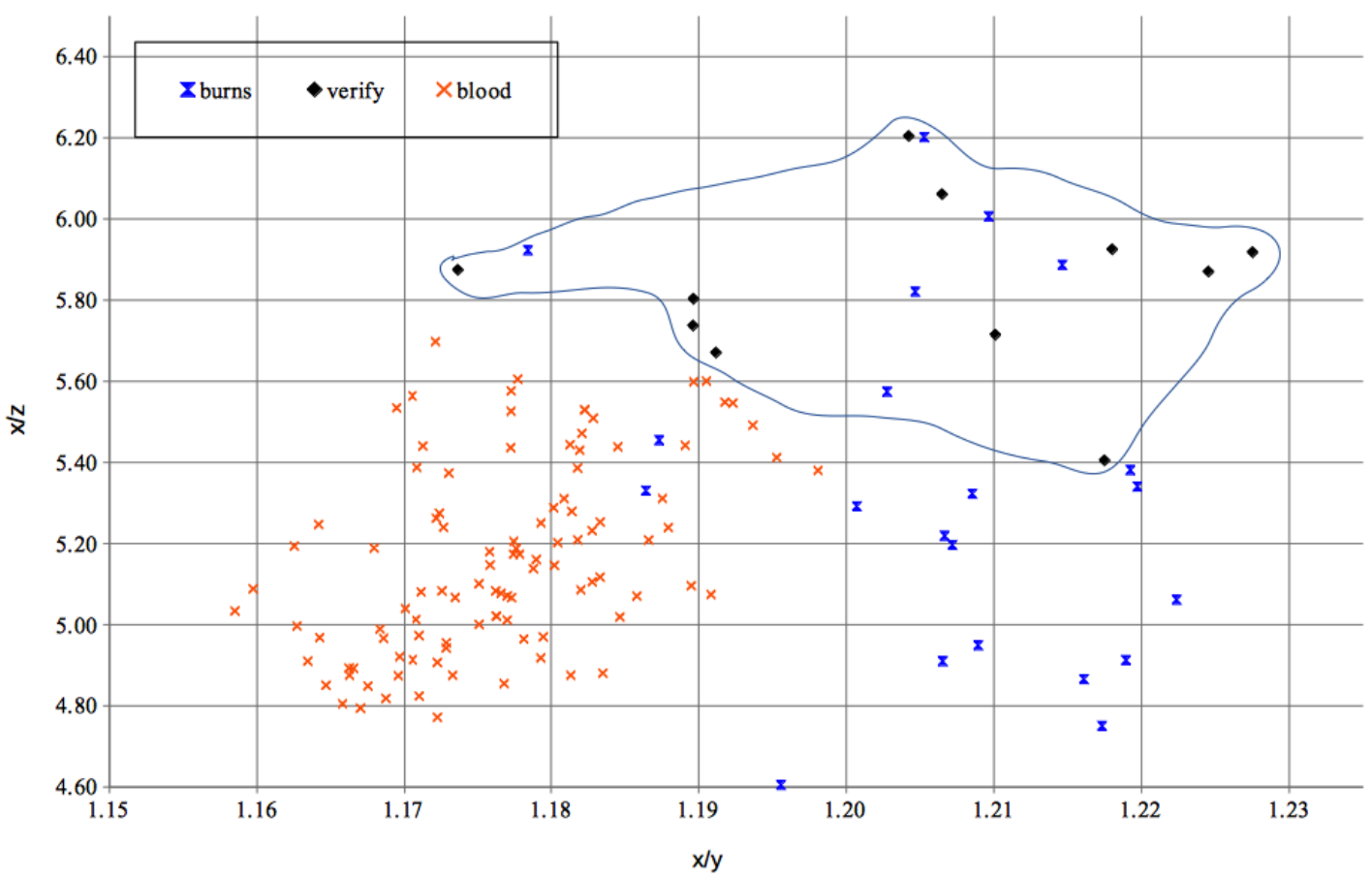

Figure 19. position of the glutes unknown stains color values in burns-blood values "cloud".

\section{Acknowledgements}

Special thanks goes to Prof. Giulio Fanti of Padua University: he stood by me in all phases of the study and provided me valuable tips and insights scientific.

My thanks also goes to my colleague Pierandrea Malfi of CRIS, for his valuable advice on the preparation of this report.

\section{References}

1. G. Fanti, R. Basso, The Turin Shroud, Optical Research in the Past Present and Future, Publisher Nova Science Pub Inc., (2008). 
2. G. Fanti, P. Malfi, The Shroud of Turin - First century After Christ!, Pan Stanford Publishing Pte. Ltd., Singapore, (2015).

3. C. Privitera, Costruzione di un'immagine quantitativa della Sindone di Torino per il riconoscimento di dettagli, graduation thesis, Padua University, (2009).

4. C. Privitera, G. Fanti, Construction of a quantitative image of the Turin Shroud for details recognition, Proc. IWSAI, ENEA, Frascati, pp. 35-45, (2010).

5. M. Artom, P. Soardo, Caratteristiche fotometriche e colorimetriche della Sindone, Proc. II Conv. Naz. di Sindonologia, Bologna 1981, CLUEB, Bologna, pp 321-329, (1983).

6. C. Oleari, Misurare il colore, Hoepli Editore, (1998).

7. D. Pascale, A Review of $R G B$ color spaces...from $x y Y$ to $R^{\prime} G^{\prime} B^{\prime}$, The Babelcolor Company, http://www.babelcolor.com/, (2002-2003),

8. R. J. Schneider, Digital Image Analysis of the Shroud of Turin: An Ongoing Investigation, da http://www.ohioshroudconference.com/papers.htm, (2008).

9. G. Fanti, Can Corona Discharge explain the body image formation of the Turin Shroud?, J. of Imaging Science and Technology, 54 No. 2, pp. 020508-1/10, (2010).

10. G. Fanti, Hypotheses regarding the formation of the body image on the Turin Shroud. A critical compendium, J. of Imaging Sci. Technol., 55 No.6, p. 060507, (2011).

11. G. Fanti, R. Basso, G. Bianchini, Turin Shroud: Compatibility Between a Digitized Body Image and a Computerized Anthropomorphous Manikin, J. of Imaging Sci. Technol., 54 No.5, p. 050503$1 / 8,(2010)$.

12. G. Fanti, J.A. Botella, P. Di Lazzaro, T. Heimburger, R. Schneider, N. Svensson, Microscopic and Macroscopic Characteristics of the Shroud of Turin Image Superficiality, J. of Imaging Sci. Technol., 54 No. 4, p. 040201-1/8, (2010).

13. E.J. Jumper, Adler A.D., Jackson J.P., Pellicori S.F., Heller J.H., Druzik J.R., "A comprehensive examination of the various stains and images on the Shroud of Turin", Archaeological Chemistry III, ACS Advances in Chemistry $n^{\circ}$ 205, J.B. Lambert, Editor, Chapter 22, American Chemical Society, Washington D.C., pp. 447-476, (1984).

14. L. A. Schwalbe, R.N. Rogers, Physics and chemistry of the Shroud of Turin, a summary of the 1978 investigation, Analytica Chimica Acta, 135, pp. 3-49, (1982).

15. Fanti, R. Maggiolo, The double superficiality of the frontal image of the Turin Shroud, J. of Optics A: Pure and Applied Optics, 6, issue 6, pages 491- 503, (2004).

16. G. Fanti, F. Lattarulo, G. Pesavento, Experimental Results Using Corona Discharge to Attempt to Reproduce the Turin Shroud Image, ATSI 2014, WOPSAS, Bari, Italy, (2014)

17. G. Fanti, R. Basso, Statistical Analysis Of Dusts Taken From Different Areas Of The Turin Shroud, Int. Conf. The Shroud Of Turin: Perspectives on A Multifaceted Enigma, Ohio State University, (2008), Libreria Progetto, Padova, Italy (2009).

18. Faccini B., Carreira E., Fanti G., De Palacios J., Villalain J., The Death Of The Shroud Man: An Improved Review, Proc. Int. Conf.: The Shroud Of Turin: Perspectives on A Multifaceted Enigma, Ohio USA (2008), Libreria Progetto, Padova, Italy (2009).

19. G. Bedon, M. Linguanotto, L. Simonato, F. Zara, Study of the bloodstains in the Shroud of Turin. International Congress, Int. Conf. The Shroud Of Turin: Perspectives on A Multifaceted Enigma, Ohio State University, (2008), Libreria Progetto, Padova, Italy (2009).

10. M. Bevilacqua, G. Fanti, M. D’Arienzo, A. Porzionato, V. Macchi, R. De Caro, How was the Turin Shroud Man crucified?, Injury, 45 Supp.6, P. S142-S148 (2014). 